\title{
A new compact high order off-step discretization for the system of 2D quasi-linear elliptic partial differential equations
}

\author{
Ranjan K Mohanty ${ }^{1 *}$ and Nikita Setia ${ }^{2}$
}

"Correspondence:
rmohanty@sau.ac.in;
mohantyranjankumar@gmail.com
1Department of Applied
Mathematics, Faculty of
Mathematics and Computer
Science, South Asian University,
Akbar Bhawan, Chanakyapuri, New
Delhi, 110021, India
Full list of author information is
available at the end of the article

available at the end of the article

\begin{abstract}
A new fourth-order difference method for solving the system of two-dimensional quasi-linear elliptic equations is proposed. The difference scheme referred to as off-step discretization is applicable directly to the singular problems and problems in polar coordinates. Also, new fourth-order methods for obtaining the first-order normal derivatives of the solution are developed. The convergence analysis of the proposed method is discussed in details. The methods are applied to many physical problems to illustrate their accuracy and efficiency.

MSC: $65 \mathrm{~N} 06$

Keywords: quasi-linear elliptic equations; fourth-order finite difference methods; convection-diffusion equation; Burger's equation; Poisson's equation in polar coordinates; Navier-Stokes equations of motion
\end{abstract}

\section{Introduction}

We consider the two-dimensional (2D) quasi-linear elliptic partial differential equation (PDE) of the type

$$
a(x, y, u) u_{x x}+b(x, y, u) u_{y y}=f\left(x, y, u, u_{x}, u_{y}\right)
$$

where $(x, y) \in R=(0,1) \times(0,1)$, with boundary $\partial R$ (see Figure 1 ), subject to the Dirichlet boundary conditions given by

$$
u(x, y)=v(x, y), \quad(x, y) \in \partial R
$$

The PDEs of the type (1) with variable coefficients model many problems of physical significance. For instance, the convection-diffusion and Burgers' equations that represent the transport phenomena, and the highly nonlinear Navier-Stokes' (N-S) equations of motion that describe the motion of fluid flow and represent the conservation of mass, momentum and energy.

We make the following assumptions about the boundary value problem (1):

(a) $a b>0$ in $R$,

(0) 2013 Mohanty and Setia; licensee Springer. This is an Open Access article distributed under the terms of the Creative Commons Attribution License (http://creativecommons.org/licenses/by/2.0), which permits unrestricted use, distribution, and reproduction in any medium, provided the original work is properly cited. 


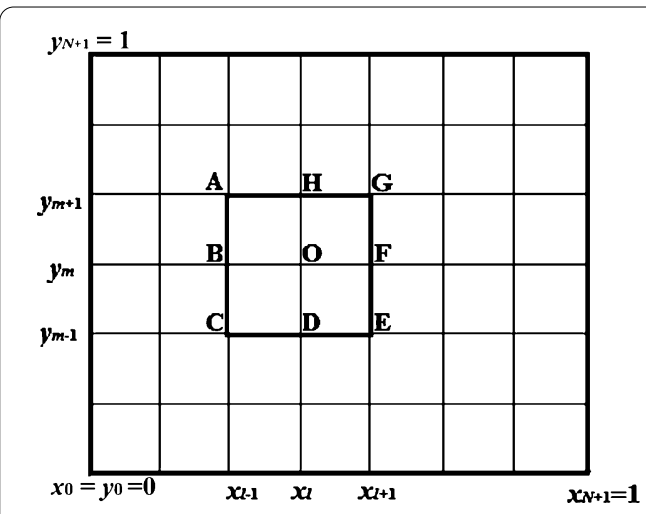

Single computational cell ACEG in the region $R \cup \partial R$

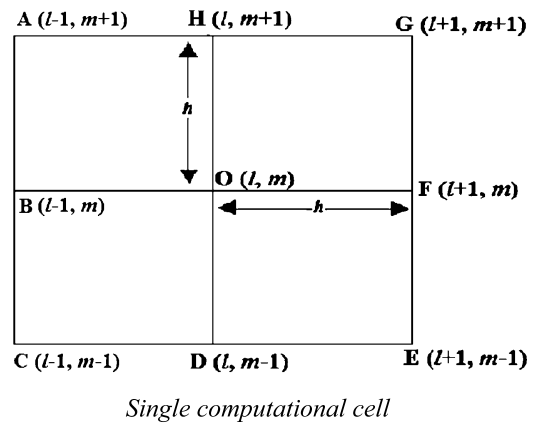

Figure 1 Schematic representation of two dimensional nine point compact cell.

(b) $u(x, y) \in C^{6}$,

(c) $a, b \in C^{4}$,

(d) $f$ is continuous,

(e) $\frac{\partial f}{\partial u} \geq 0$,

(f) $\left|\frac{\partial f}{\partial u_{x}}\right| \leq H$,

(g) $\left|\frac{\partial f}{\partial u_{y}}\right| \leq I$,

where $H$ and $I$ are positive constants and $C^{m}$ is the set of all functions of $x$ and $y$ with continuous partial derivatives up to order $m$ in the region $R$. The condition (a) guarantees the ellipticity of equation (1). Conditions (e), (f) and (g) are the necessary conditions for the existence and uniqueness of the solution of boundary value problem (1)-(2) (see [1]).

A number of high order compact schemes have been reported for the linear elliptic problems like Poisson's equation and the convection diffusion equation (see [2-9]). Ananthakrishnaiah and Saldanha [10] framed a 13-point fourth-order compact scheme for the solution of a scalar nonlinear elliptic PDE, which was later extended to a system of equations by Saldanha [11]. The finite difference methods for solving the steady state incompressible $\mathrm{N}-\mathrm{S}$ equations vary considerably in terms of accuracy and efficiency. It has been discovered that although central difference approximations are locally second-order accurate, they often suffer from computational instability and the resulting solutions exhibit non-physical oscillations. The upwind difference approximations, though computationally stable, are only first-order accurate and the resulting solutions exhibit the effects of artificial viscosity. A number of high order compact schemes for the solution of the $\mathrm{N}-\mathrm{S}$ equations in stream function vorticity form in the Cartesian coordinates were proposed in [12-15]. In 1997, Mohanty [16] proposed fourth-order difference methods for 2D nonlinear elliptic boundary value problems with variable coefficients using only nine grid points of a single computational cell. This method could be successfully applied to the N-S model equations in polar coordinates. Later, Mohanty and Dey [17] developed the fourth-order accurate estimates of the first-order normal derivatives of the solution $v i z(\partial u / \partial n)$. However, the methods [16] and [17] could not be directly applied to singular elliptic problems, and they required suitable modifications at the points of singularity. In this regard, Mohanty and Singh [18] derived an off-step fourth-order discretization for the solution of singularly perturbed two-dimensional nonlinear elliptic problems and the estimates of $(\partial u / \partial n)$, which were directly applicable to singular elliptic problems. 
In this article, we develop new off-step fourth-order discretizations for the solution of the system of quasi-linear elliptic PDEs with variable coefficients, and the estimates of $(\partial u / \partial n)$, using the nine grid points of a single computational cell (see Figure 1). The main advantage of the proposed methods is that they are directly applicable to the singular problems and the problems in polar coordinates, without any need of modifications, hence reducing the manual and mechanical calculations reasonably.

An outline of the article is as follows: In Section 2, we discuss and derive the off-step fourth-order compact discretization schemes for the solution of a nonlinear elliptic equation with variable coefficients and the estimates of $(\partial u / \partial n)$. These methods are further extended to the solution of the quasi-linear PDE given by (1)-(2). In Section 3, we establish the fourth-order convergence of the method for a scalar equation under appropriate conditions. Further, in Section 4, the stability analysis of the steady state convection diffusion equation is conducted. In Section 5, we generalize our methods for the system of quasi-linear PDEs with variable coefficients, subject to the Dirichlet boundary conditions. In Section 6, we implement the proposed methods over linear and nonlinear problems of physical significance to illustrate and examine the accuracy of these methods. Section 7 contains some concluding remarks about this article.

\section{The off-step discretization and derivation}

We first consider the following two-dimensional nonlinear elliptic PDE:

$$
a(x, y) u_{x x}+b(x, y) u_{y y}=f\left(x, y, u, u_{x}, u_{y}\right)
$$

for $(x, y) \in R$, subject to the Dirichlet boundary conditions given by (2).

We superimpose on the domain $R$ a rectangular grid with spacing $h>0$ in both $x$ and $y$-directions. Let us introduce the following notations:

(a) Each grid point is given by $\left(x_{l}, y_{m}\right)$ or simply $(l, m)$ for $x_{l}=l h$ and $y_{m}=m h$, $0 \leq l, m \leq N+1$, where $(N+1) h=1$.

Further, at each grid point $(l, m)$, let:

(b) $U_{l, m}$ and $u_{l, m}$ denote the exact and approximate values of $u\left(x_{l}, y_{m}\right)$, respectively.

(c) $f_{l, m}=f\left(x_{l}, y_{m}, U_{l, m}, U_{x l, m}, U_{y l, m}\right)$.

(d) For $S=a, b, U, \alpha, \beta$ and $\gamma$, let

$$
S_{p q}=\frac{\partial^{p+q} S}{\partial x^{p} \partial y^{q}}, \quad p, q=0,1,2, \ldots
$$

Then, for $0 \leq l, m \leq N+1$, differential equation (3) can be written as

$$
a_{00} U_{20}+b_{00} U_{02}=f_{l, m}
$$

For the fourth-order discretization of PDE (3), we simply follow the approach given by Chawla and Shivakumar [19].

We set the following approximations:

$$
\begin{aligned}
& \bar{U}_{l \pm \frac{1}{2}, m}=\left(U_{l \pm 1, m}+U_{l, m}\right) / 2, \\
& \bar{U}_{l, m \pm \frac{1}{2}}=\left(U_{l, m \pm 1}+U_{l, m}\right) / 2,
\end{aligned}
$$




$$
\begin{aligned}
& \bar{U}_{x l, m}=\left(U_{l+1, m}-U_{l-1, m}\right) /(2 h), \\
& \bar{U}_{x l \pm \frac{1}{2}, m}=\left( \pm U_{l \pm 1, m} \mp U_{l, m}\right) /(h), \\
& \bar{U}_{x l, m \pm \frac{1}{2}}=\left(U_{l+1, m \pm 1}-U_{l-1, m \pm 1}+U_{l+1, m}-U_{l-1, m}\right) /(4 h), \\
& \bar{U}_{y l, m}=\left(U_{l, m+1}-U_{l, m-1}\right) /(2 h), \\
& \bar{U}_{y l \pm \frac{1}{2}, m}=\left(U_{l \pm 1, m+1}-U_{l \pm 1, m-1}+U_{l, m+1}-U_{l, m-1}\right) /(4 h), \\
& \bar{U}_{y l, m \pm \frac{1}{2}}=\left( \pm U_{l, m \pm 1} \mp U_{l, m}\right) /(h), \\
& \bar{U}_{x x l, m}=\left(U_{l+1, m}-2 U_{l, m}+U_{l-1, m}\right) /\left(h^{2}\right), \\
& \bar{U}_{x x l, m \pm 1}=\left(U_{l+1, m \pm 1}-2 U_{l, m \pm 1}+U_{l-1, m \pm 1}\right) /\left(h^{2}\right), \\
& \bar{U}_{y y l, m}=\left(U_{l, m+1}-2 U_{l, m}+U_{l, m-1}\right) /\left(h^{2}\right), \\
& \bar{U}_{y y l \pm 1, m}=\left(U_{l \pm 1, m+1}-2 U_{l \pm 1, m}+U_{l \pm 1, m-1}\right) /\left(h^{2}\right) .
\end{aligned}
$$

Define

$$
\begin{aligned}
& \bar{f}_{l \pm \frac{1}{2}, m}=f\left(x_{l \pm \frac{1}{2}}, y_{m}, \bar{U}_{l \pm \frac{1}{2}, m}, \bar{U}_{x l \pm \frac{1}{2}, m}, \bar{U}_{y l \pm \frac{1}{2}, m}\right) \\
& \bar{f}_{l, m \pm \frac{1}{2}}=f\left(x_{l}, y_{m \pm \frac{1}{2}}, \bar{U}_{l, m \pm \frac{1}{2}}, \bar{U}_{x l, m \pm \frac{1}{2}}, \bar{U}_{y l, m \pm \frac{1}{2}}\right)
\end{aligned}
$$

Let

$$
\begin{aligned}
\overline{\bar{U}}_{l, m}= & U_{l, m}+p_{1} h^{2}\left(\bar{f}_{l+\frac{1}{2}, m}+\bar{f}_{l-\frac{1}{2}, m}\right)+p_{2} h^{2}\left(\bar{f}_{l, m+\frac{1}{2}}+\bar{f}_{l, m-\frac{1}{2}}\right) \\
& +p_{3} h^{2} \bar{U}_{x x l, m}+p_{4} h^{2} \bar{U}_{y y l, m}, \\
\overline{\bar{U}}_{x l, m}= & \bar{U}_{x l, m}+q_{1} h\left(\bar{f}_{l+\frac{1}{2}, m}-\bar{f}_{l-\frac{1}{2}, m}\right)+q_{2} h\left(\bar{U}_{y y l+1, m}-\bar{U}_{y y l-1, m}\right) \\
& +q_{3} h^{2} \bar{U}_{x x l, m}+q_{4} h^{2} \bar{U}_{y y l, m}, \\
\overline{\bar{U}}_{y l, m}= & \bar{U}_{y l, m}+r_{1} h\left(\bar{f}_{l, m+\frac{1}{2}}-\bar{f}_{l, m-\frac{1}{2}}\right)+r_{2} h\left(\bar{U}_{x x l, m+1}-\bar{U}_{x x l, m-1}\right) \\
& +r_{3} h^{2} \bar{U}_{x x l, m}+r_{4} h^{2} \bar{U}_{y y l, m},
\end{aligned}
$$

where $p_{k} \mathrm{~s}, q_{k} \mathrm{~s}$ and $r_{k} \mathrm{~s}(1 \leq k \leq 4)$ are the parameters to be suitably determined.

Finally, define

$$
\overline{\bar{f}}_{l, m}=f\left(x_{l}, y_{m}, \overline{\bar{U}}_{l, m}, \overline{\bar{U}}_{x l, m}, \overline{\bar{U}}_{y l, m}\right)
$$

Then, at each internal grid point $(l, m)$, differential equation (3) is discretized by

$$
\begin{aligned}
L[U] \equiv & {\left[I_{1} \delta_{x}^{2}+I_{2} \delta_{y}^{2}+I_{3}\left(2 \delta_{x}^{2} \mu_{y} \delta_{y}\right)+I_{4}\left(2 \delta_{y}^{2} \mu_{x} \delta_{x}\right)+I_{5}\left(\delta_{x}^{2} \delta_{y}^{2}\right)\right] U_{l, m} } \\
= & h^{2}\left[J_{1} \bar{f}_{l+\frac{1}{2}, m}+J_{2} \bar{f}_{l-\frac{1}{2}, m}+J_{3} \bar{f}_{l, m+\frac{1}{2}}+J_{4} \bar{f}_{l, m-\frac{1}{2}}-2 \overline{\bar{f}}_{l, m}\right] \\
& +\bar{T}_{l, m} ; \quad 1 \leq l, m \leq N
\end{aligned}
$$


for $\bar{T}_{l, m}=O\left(h^{6}\right)$, where we denote

$$
\begin{aligned}
& K_{1}=a_{10} / a_{00}, \quad K_{2}=b_{01} / b_{00}, \\
& I_{1}=6 a_{00}+\frac{1}{2} h^{2}\left(a_{20}+a_{02}-2 K_{1} a_{10}-2 K_{2} a_{01}\right), \\
& I_{2}=6 b_{00}+\frac{1}{2} h^{2}\left(b_{20}+b_{02}-2 K_{1} b_{10}-2 K_{2} b_{01}\right), \\
& I_{3}=\frac{1}{2} h\left(a_{01}-K_{2} a_{00}\right), \quad I_{4}=\frac{1}{2} h\left(b_{10}-K_{1} b_{00}\right), \quad I_{5}=\frac{1}{2}\left(a_{00}+b_{00}\right), \\
& J_{1}=2-h K_{1}, \quad J_{2}=2+h K_{1}, \quad J_{3}=2-h K_{2}, \quad J_{4}=2+h K_{2}
\end{aligned}
$$

for $\delta_{x} U_{l}=\left(U_{l+1 / 2}-U_{l-1 / 2}\right)$ and $\mu_{x} U_{l}=\frac{1}{2}\left(U_{l+1 / 2}+U_{l-1 / 2}\right)$ being the central and average difference operators in $x$-direction etc.

Now, with the help of Taylor series expansion, it is easy to obtain

$$
\left.L[U]=h^{2} J_{1} f_{l+\frac{1}{2}, m}+J_{2} f_{l-\frac{1}{2}, m}+J_{3} f_{l, m+\frac{1}{2}}+J_{4} f_{l, m-\frac{1}{2}}-2 f_{l, m}\right]+O\left(h^{6}\right), \quad 1 \leq l, m \leq N .
$$

Now, let

$$
\alpha=\frac{\partial f}{\partial U}, \quad \beta=\frac{\partial f}{\partial U_{x}}, \quad \gamma=\frac{\partial f}{\partial U_{y}} .
$$

Using (6.1) and (6.2) and simplifying (5.1)-(5.8) by Taylor series expansions, we obtain

$$
\begin{aligned}
& \bar{f}_{l \pm \frac{1}{2}, m}=f_{l \pm \frac{1}{2}, m}+\frac{h^{2}}{24} T_{1}+O\left( \pm h^{3}+h^{4}\right) \\
& \bar{f}_{l, m \pm \frac{1}{2}}=f_{l, m \pm \frac{1}{2}}+\frac{h^{2}}{24} T_{2}+O\left( \pm h^{3}+h^{4}\right)
\end{aligned}
$$

where

$$
\begin{aligned}
& T_{1}=3 U_{20} \alpha_{00}+U_{30} \beta_{00}+\left(3 U_{21}+4 U_{03}\right) \gamma_{00}, \\
& T_{2}=3 U_{02} \alpha_{00}+\left(4 U_{30}+3 U_{12}\right) \beta_{00}+U_{03} \gamma_{00} .
\end{aligned}
$$

Using equations (11.1), (11.2), simplifying (5.9)-(5.12) and (7.1)-(7.3), we obtain

$$
\begin{aligned}
& \overline{\bar{U}}_{l, m}=U_{l, m}+\frac{h^{2}}{6} T_{3}+O\left(h^{4}\right), \\
& \overline{\bar{U}}_{x l, m}=U_{x l, m}+\frac{h^{2}}{6} T_{4}+O\left(h^{4}\right), \\
& \overline{\bar{U}}_{y l, m}=U_{y l, m}+\frac{h^{2}}{6} T_{5}+O\left(h^{4}\right),
\end{aligned}
$$

where

$$
\begin{aligned}
& T_{3}=\left[12\left(p_{1}+p_{2}\right) a_{00}+6 p_{3}\right] U_{20}+\left[12\left(p_{1}+p_{2}\right) b_{00}+6 p_{4}\right] U_{02}, \\
& T_{4}=\left(1+6 q_{1} a_{00}\right) U_{30}+6\left(q_{1} a_{10}+q_{3}\right) U_{20}+6\left(q_{1} b_{00}+2 q_{2}\right) U_{12}+6\left(q_{1} b_{10}+q_{4}\right) U_{02}, \\
& T_{5}=\left(1+6 r_{1} b_{00}\right) U_{03}+6\left(r_{1} a_{01}+r_{3}\right) U_{20}+6\left(r_{1} a_{00}+2 r_{2}\right) U_{21}+6\left(r_{1} b_{01}+r_{4}\right) U_{02} .
\end{aligned}
$$


Finally, from (8), using (12.1)-(12.3), we obtain

$$
\overline{\bar{f}}_{l, m}=f_{l, m}+\frac{h^{2}}{6} T_{6}+O\left(h^{4}\right)
$$

where

$$
T_{6}=T_{3} \alpha_{00}+T_{4} \beta_{00}+T_{5} \gamma_{00} .
$$

Substituting approximations (11.1), (11.2) and (13) into (9), and by the help of (10), we obtain

$$
\bar{T}_{l, m}=\frac{h^{4}}{6}\left(2 T_{6}-T_{1}-T_{2}\right)+O\left(h^{6}\right) .
$$

Thus, for the proposed difference method (9) to be of fourth order, the coefficient of $h^{4}$ in (14) must be zero, and hence we have

$$
T_{1}+T_{2}-2 T_{6}=0
$$

Equating to zero the coefficients of each of $\alpha_{00}, \beta_{00}$ and $\gamma_{00}$, we obtain the values of the unknown parameters as follows:

$$
\begin{aligned}
& p_{1}=1 /\left(16 a_{00}\right), \quad p_{2}=1 /\left(16 b_{00}\right), \quad p_{3}=\left(1-a_{00} / b_{00}\right) / 8, \quad p_{4}=\left(1-b_{00} / a_{00}\right) / 8, \\
& q_{1}=1 /\left(4 a_{00}\right), \quad q_{2}=\left(1-b_{00} / a_{00}\right) / 8, \quad q_{3}=-a_{10} /\left(4 a_{00}\right), \quad q_{4}=-b_{10} /\left(4 a_{00}\right), \\
& r_{1}=1 /\left(4 b_{00}\right), \quad r_{2}=\left(1-a_{00} / b_{00}\right) / 8, \quad r_{3}=-a_{01} /\left(4 b_{00}\right), \quad r_{4}=-b_{01} /\left(4 b_{00}\right)
\end{aligned}
$$

thereby reducing $\bar{T}_{l, m}$ to $O\left(h^{6}\right)$. Thus the difference method of $O\left(h^{4}\right)$ for nonlinear PDE (3) is given by (9) for the above values of parameters.

Now, we consider the numerical method of $O\left(h^{4}\right)$ for the solution of 2D quasi-linear elliptic equation (1). In order to understand the concept to develop the method for the quasi-linear case, we consider the following differential equation:

$$
u^{\prime \prime}=f(x), \quad 0<x<1 .
$$

A fourth-order method for differential equation (15) is given by

$$
U_{l-1}-2 U_{l}+U_{l+1}=\frac{h^{2}}{12}\left[f_{l}+h^{2} f_{x x l}\right]+O\left(h^{6}\right)
$$

where $U_{l}=u\left(x_{l}\right), f_{l}=f\left(x_{l}\right)$ and $f_{x x l}=\frac{\partial^{2} f_{l}}{\partial x^{2}}$.

Whenever the differential equation (15) is of the form $u^{\prime \prime}=f(x, u)$, the evaluation of $f_{x x}$ is difficult and formula (16) needs to be modified. Substituting $h^{2} f_{x x l}=f_{l+1}-2 f_{l}+f_{l-1}+O\left(h^{4}\right)$ in (16), we obtain the modified version of (16) due to Numerov as

$$
U_{l-1}-2 U_{l}+U_{l+1}=\frac{h^{2}}{12}\left[f_{l+1}+f_{l-1}+10 f_{l}\right]+O\left(h^{6}\right)
$$

where $f_{l}=f\left(x_{l}, U_{l}\right)$. Note that $(17)$ is consistent with the differential equation $u^{\prime \prime}=f(x, u)$. 
Now, we use the above concept to derive the numerical method for quasi-linear equation (1). Since the coefficients are the functions of not only the independent variables $x$ and $y$ but also of the dependent variable $u$, i.e., $a=a(x, y, u)$ and $b=b(x, y, u)$, the difference scheme (9) cannot be applied directly as the first- and second-order derivatives of $u$ are unknown at the internal grid points. Thus further discretizations of $u_{x}, u_{y}, u_{x x}$ and $u_{y y}$ are required in the method (9) without affecting its order. For this purpose, for $S=a$ and $b$, we use the following central differences:

$$
\begin{aligned}
& S_{10}=\left(S_{l+1, m}-S_{l-1, m}\right) / 2 h+O\left(h^{2}\right), \\
& S_{01}=\left(S_{l, m+1}-S_{l, m-1}\right) / 2 h+O\left(h^{2}\right), \\
& S_{20}=\left(S_{l+1, m}-2 S_{00}+S_{l-1, m}\right) / h^{2}+O\left(h^{2}\right), \\
& S_{02}=\left(S_{l, m+1}-2 S_{00}+S_{l, m-1}\right) / h^{2}+O\left(h^{2}\right),
\end{aligned}
$$

where

$$
\begin{aligned}
& S_{00}=S\left(x_{l}, y_{m}, U_{l, m}\right), \\
& S_{l \pm 1, m}=S\left(x_{l \pm 1}, y_{m}, U_{l \pm 1, m}\right), \\
& S_{l, m \pm 1}=S\left(x_{l}, y_{m \pm 1}, U_{l, m \pm 1}\right) .
\end{aligned}
$$

Upon substitution of the central differences (18.1)-(18.4) in the method (9), it is easy to verify that

$$
\begin{aligned}
I_{1}= & 4 a_{00}+\frac{1}{2}\left[a_{l+1, m}+a_{l-1, m}+a_{l, m+1}+a_{l, m-1}\right] \\
& -\frac{\left(a_{l, m+1}-a_{l, m-1}\right)\left(b_{l, m+1}-b_{l, m-1}\right)}{4 b_{00}}-\frac{\left(a_{l+1, m}-a_{l-1, m}\right)^{2}}{4 a_{00}}+O\left(h^{4}\right), \\
I_{2}= & 4 b_{00}+\frac{1}{2}\left[b_{l+1, m}+b_{l-1, m}+b_{l, m+1}+b_{l, m-1}\right] \\
& -\frac{\left(a_{l+1, m}-a_{l-1, m}\right)\left(b_{l+1, m}-b_{l-1, m}\right)}{4 a_{00}}-\frac{\left(b_{l, m+1}-b_{l, m-1}\right)^{2}}{4 b_{00}}+O\left(h^{4}\right), \\
I_{3}= & \frac{\left(a_{l, m+1}-a_{l, m-1}\right)}{4}-\left(\frac{b_{l, m+1}-b_{l, m-1}}{4 b_{00}}\right) a_{00}+O\left(h^{3}\right), \\
I_{4}= & \frac{\left(b_{l+1, m}-b_{l-1, m}\right)}{4}-\left(\frac{a_{l+1, m}-a_{l-1, m}}{4 a_{00}}\right) b_{00}+O\left(h^{3}\right) .
\end{aligned}
$$

We observe that the truncation error $\bar{T}_{l, m}$ retains its order $O\left(h^{6}\right)$, and hence we obtain the required numerical method of $O\left(h^{4}\right)$ for the solution of quasi-linear elliptic PDE (1).

After having determined the fourth-order approximations to the solution of equation (3), we now discuss the fourth-order numerical methods for the estimates of $(\partial u / \partial x)$ and $(\partial u / \partial y)$. One may compute these values using the standard central differences:

$$
\begin{aligned}
& u_{x l, m}=\left(u_{l+1, m}-u_{l-1, m}\right) /(2 h), \\
& u_{y l, m}=\left(u_{l, m+1}-u_{l, m-1}\right) /(2 h) .
\end{aligned}
$$


It is found that the standard central differences (19.1) and (19.2) yield second-order accurate results irrespective of whether fourth-order difference method (9) or standard difference scheme is used to solve PDE (3). Thus, new difference methods for computing the numerical values of $(\partial u / \partial x)$ and $(\partial u / \partial y)$ are developed, which are found to yield $O\left(h^{4}\right)$ accurate results when used in conjunction with the nine-point formula (9).

At each grid point $(l, m)$, we denote the exact and the approximate solutions of $(\partial u / \partial x)$, $(\partial u / \partial y)$ by $U_{x l, m}, U_{y l, m}$ and $u_{x l, m}, u_{y l, m}$, respectively. Then, following the techniques given by Stephenson [20], for $l, m=1(1) N$, we obtain

$$
\begin{aligned}
U_{x l, m}= & \frac{1}{2 h}\left(2 \mu_{x} \delta_{x}\right) U_{l, m}+\frac{1}{6 a_{00}}\left(a_{10} \delta_{x}^{2}+b_{10} \delta_{y}^{2}\right) U_{l, m}+\frac{b_{00}}{12 h a_{00}}\left(2 \delta_{y}^{2} \mu_{x} \delta_{x}\right) U_{l, m} \\
& -\frac{h}{6 a_{00}}\left(\bar{f}_{l+\frac{1}{2}, m}-\bar{f}_{l-\frac{1}{2}, m}\right)+\bar{T}_{l, m}^{(x)}, \\
U_{y l, m}= & \frac{1}{2 h}\left(2 \mu_{y} \delta_{y}\right) U_{l, m}+\frac{1}{6 b_{00}}\left(a_{01} \delta_{x}^{2}+b_{01} \delta_{y}^{2}\right) U_{l, m}+\frac{a_{00}}{12 h b_{00}}\left(2 \delta_{x}^{2} \mu_{y} \delta_{y}\right) U_{l, m} \\
& -\frac{h}{6 b_{00}}\left(\bar{f}_{l, m+\frac{1}{2}}-\bar{f}_{l, m-\frac{1}{2}}\right)+\bar{T}_{l, m}^{(y)} .
\end{aligned}
$$

A simple Taylor series expansion would yield

$$
\begin{aligned}
U_{x l, m}= & \frac{1}{2 h}\left(2 \mu_{x} \delta_{x}\right) U_{l, m}+\frac{1}{6 a_{00}}\left(a_{10} \delta_{x}^{2}+b_{10} \delta_{y}^{2}\right) U_{l, m}+\frac{b_{00}}{12 h a_{00}}\left(2 \delta_{y}^{2} \mu_{x} \delta_{x}\right) U_{l, m} \\
& -\frac{h}{6 a_{00}}\left(f_{l+\frac{1}{2}, m}-f_{l-\frac{1}{2}, m}\right)+O\left(h^{4}\right) .
\end{aligned}
$$

Then, using equations (11.1) and (21) in (20.1), we obtain $\bar{T}_{l, m}^{(x)}=O\left(h^{4}\right)$. Similarly, we obtain $\bar{T}_{l, m}^{(y)}=O\left(h^{4}\right)$. Hence, equations (20.1)-(20.2) give the fourth-order approximation to the first-order normal derivatives of the solution of nonlinear equation (3). The numerical methods (20.1)-(20.2) are applicable when the fourth-order numerical solutions of $u$ are known at each internal grid point. Further, the Dirichlet boundary conditions are given by (2). The difference method (9) for the determination of $u$ can be easily expressed in triblock-diagonal matrix form, and the methods (20.1)-(20.2) for determination of $(\partial u / \partial x)$ and $(\partial u / \partial y)$ can be expressed in diagonal matrices form, thus can be easily solved. The proposed methods (9), (20.1) and (20.2) are directly applicable to singular elliptic problems in the region $R$.

Now, for the two-dimensional quasi-linear elliptic equation (1), using the approximations (18.1) and (18.2) in (20.1)-(20.2), we easily obtain

$$
\begin{aligned}
U_{x l, m}= & \frac{1}{2 h}\left(2 \mu_{x} \delta_{x}\right) U_{l, m}+\frac{1}{12 h a_{00}}\left(\left(a_{l+1, m}-a_{l-1, m}\right) \delta_{x}^{2}+\left(b_{l+1, m}-b_{l-1, m}\right) \delta_{y}^{2}\right) U_{l, m} \\
& +\frac{b_{00}}{12 h a_{00}}\left(2 \mu_{x} \delta_{x}\right) \delta_{y}^{2} U_{l, m}-\frac{h}{6 a_{00}}\left(\bar{f}_{l+\frac{1}{2}, m}-\bar{f}_{l-\frac{1}{2}, m}\right)+\bar{T}_{l, m}^{(x)}, \quad 1 \leq l, m \leq N, \\
U_{y l, m}= & \frac{1}{2 h}\left(2 \mu_{y} \delta_{y}\right) U_{l, m}+\frac{1}{12 h b_{00}}\left(\left(a_{l, m+1}-a_{l, m-1}\right) \delta_{x}^{2}+\left(b_{l, m+1}-b_{l, m-1}\right) \delta_{y}^{2}\right) U_{l, m} \\
& +\frac{a_{00}}{12 h b_{00}}\left(2 \mu_{y} \delta_{y}\right) \delta_{x}^{2} U_{l, m}-\frac{h}{6 b_{00}}\left(\bar{f}_{l, m+\frac{1}{2}}-\bar{f}_{l, m-\frac{1}{2}}\right)+\bar{T}_{l, m}^{(y)}, \quad 1 \leq l, m \leq N,
\end{aligned}
$$

where $\bar{T}_{l, m}^{(x)}$ and $\bar{T}_{l, m}^{(y)}$ are of $O\left(h^{4}\right)$. 


\section{Convergence analysis}

We consider the 2D nonlinear elliptic partial differential equation

$$
A u_{x x}+B u_{y y}=f\left(x, y, u, u_{x}, u_{y}\right)
$$

defined in the region $R$, subject to $u(x, y)=v(x, y),(x, y) \in \partial R$, where $A, B>0$ are constants.

Then the difference method (9) for equation (23) is given by

$$
\begin{aligned}
& {\left[6 A \delta_{x}^{2}+6 B \delta_{y}^{2}+\frac{(A+B)}{2} \delta_{x}^{2} \delta_{y}^{2}\right] u_{l, m}} \\
& \quad=2 h^{2}\left[\bar{f}_{l+\frac{1}{2}, m}+\bar{f}_{l-\frac{1}{2}, m}+\bar{f}_{l, m+\frac{1}{2}}+\bar{f}_{l, m-\frac{1}{2}}-\overline{\bar{f}}_{l, m}\right] ; \quad 1 \leq l, m \leq N
\end{aligned}
$$

Let, for each $(l, m)$ such that $1 \leq l, m \leq N$,

$$
M_{l, m}=2 h^{2}\left[\bar{f}_{l+\frac{1}{2}, m}+\bar{f}_{l-\frac{1}{2}, m}+\bar{f}_{l, m+\frac{1}{2}}+\bar{f}_{l, m-\frac{1}{2}}-\overline{\bar{f}}_{l, m}\right]+\text { boundary values }
$$

and $E_{l, m}=u_{l, m}-U_{l, m}$.

Also, for $S=M, u, U, \bar{T}$ and $E$, let

$$
S=\left[S_{1,1}, S_{2,1}, \ldots, S_{N, 1}, S_{1,2}, S_{2,2}, \ldots, S_{N, 2}, \ldots, S_{1, N}, S_{2, N}, \ldots, S_{N, N}\right]_{N^{2} \times 1}^{\mathrm{t}}
$$

where $t$ denotes the transpose of the matrix.

Then, varying $(l, m)$ such that $1 \leq l, m \leq N$, equation (24) may be written in the matrix form as

$$
\mathbf{D u}+\mathbf{M}(\mathbf{u})=\mathbf{0}
$$

where

$$
\mathbf{D}=\left[\begin{array}{lll}
\mathbf{K} & \mathbf{R} & \mathbf{K}
\end{array}\right]_{N^{2} \times N^{2}} \quad \text { (Tri-block diagonal matrix) }
$$

for

$$
\mathbf{R}=\left[\begin{array}{lll}
-5 A+B & 10(A+B) & -5 A+B
\end{array}\right]_{N \times N} \quad \text { (Tri-diagonal matrix) }
$$

and

$$
\mathbf{K}=\left[\begin{array}{lll}
-(A+B) / 2 & -5 B+A & -(A+B) / 2
\end{array}\right]_{N \times N} \quad \text { (Tri-diagonal matrix). }
$$

We assume here that $B<5 A$ and $A<5 B$. Thus, all the diagonal entries of matrix $\mathbf{D}$ are positive and all the off-diagonal entries are negative.

Since $\mathbf{U}$ is the exact solution vector, we have

$$
\mathbf{D U}+\mathbf{M}(\mathbf{U})+\overline{\mathbf{T}}=\mathbf{0},
$$

where $\bar{T}_{l, m}=O\left(h^{6}\right)$ for each $(l, m)$ such that $1 \leq l, m \leq N$. 
Now, let

$$
\begin{aligned}
& \hat{f}_{l \pm \frac{1}{2}, m}=f\left(x_{l \pm \frac{1}{2}}, y_{m}, \bar{u}_{l \pm \frac{1}{2}, m}, \bar{u}_{x l \pm \frac{1}{2}, m}, \bar{u}_{y l \pm \frac{1}{2}, m}\right) \simeq \bar{f}_{l \pm \frac{1}{2}, m} \\
& \hat{f}_{l, m \pm \frac{1}{2}}=f\left(x_{l}, y_{m \pm \frac{1}{2}}, \bar{u}_{l, m \pm \frac{1}{2}}, \bar{u}_{x l, m \pm \frac{1}{2}}, \bar{u}_{y l, m \pm \frac{1}{2}}\right) \simeq \bar{f}_{l, m \pm \frac{1}{2}} \\
& \tilde{f}_{l, m}=f\left(x_{l}, y_{m}, \overline{\bar{u}}_{l, m}, \overline{\bar{u}}_{x l, m}, \overline{\bar{u}}_{y l, m}\right) \simeq \overline{\bar{f}}_{l, m} .
\end{aligned}
$$

We may write

$$
\begin{aligned}
& \hat{f}_{l \pm \frac{1}{2}, m}-\bar{f}_{l \pm \frac{1}{2}, m}=\left(\bar{u}_{l \pm \frac{1}{2}, m}-\bar{U}_{l \pm \frac{1}{2}, m}\right) G_{l \pm \frac{1}{2}, m}^{(1)}+\left(\bar{u}_{x l \pm \frac{1}{2}, m}-\bar{U}_{x l \pm \frac{1}{2}, m}\right) H_{l \pm \frac{1}{2}, m}^{(1)} \\
&+\left(\bar{u}_{y l \pm \frac{1}{2}, m}-\bar{U}_{y l \pm \frac{1}{2}, m}\right) I_{l \pm \frac{1}{2}, m}^{(1)} \\
& \hat{f}_{l, m \pm \frac{1}{2}}-\bar{f}_{l, m \pm \frac{1}{2}}=\left(\bar{u}_{l, m \pm \frac{1}{2}}-\bar{U}_{l, m \pm \frac{1}{2}}\right) G_{l, m \pm \frac{1}{2}}^{(2)}+\left(\bar{u}_{x l, m \pm \frac{1}{2}}-\bar{U}_{x l, m \pm \frac{1}{2}}\right) H_{l, m \pm \frac{1}{2}}^{(2)} \\
&+\left(\bar{u}_{y l, m \pm \frac{1}{2}}-\bar{U}_{y l, m \pm \frac{1}{2}}\right) I_{l, m \pm \frac{1}{2}}^{(2)}, \\
& \tilde{f}_{l, m}-\overline{\bar{f}}_{l, m}=\left(\overline{\bar{u}}_{l, m}-\overline{\bar{U}}_{l, m}\right) G_{l, m}^{(3)}+\left(\overline{\bar{u}}_{x l, m}-\overline{\bar{U}}_{x l, m}\right) H_{l, m}^{(3)}+\left(\overline{\bar{u}}_{l l, m}-\overline{\bar{U}}_{y l, m}\right) I_{l, m}^{(3)}
\end{aligned}
$$

for suitable $Q_{l \pm \frac{1}{2}, m}^{(1)}, Q_{l, m \pm \frac{1}{2}}^{(2)}$ and $Q_{l, m}^{(3)}$, where $Q=G, H$ and $I$.

Also, for $Q=H$ and $I$, we may write

$$
\begin{aligned}
& Q_{l \pm \frac{1}{2}, m}^{(1)}=Q_{l, m}^{(1)} \pm \frac{h}{2} Q_{x l, m}^{(1)}+O\left(h^{2}\right), \\
& Q_{l, m \pm \frac{1}{2}}^{(2)}=Q_{l, m}^{(2)} \pm \frac{h}{2} Q_{y l, m}^{(2)}+O\left(h^{2}\right)
\end{aligned}
$$

and

$$
\begin{aligned}
& G_{l \pm \frac{1}{2}, m}^{(1)}=G_{l, m}^{(1)} \pm O(h), \\
& G_{l, m \pm \frac{1}{2}}^{(2)}=G_{l, m}^{(2)} \pm O(h) .
\end{aligned}
$$

With the help of equations (27.1)-(27.3) and (28.1)-(28.4), we obtain

$$
\mathbf{M}(\mathbf{u})-\mathbf{M}(\mathbf{U})=\mathbf{P E},
$$

where $\mathbf{P}=\left(P_{r, s}\right)\left[1 \leq r \leq N^{2}, 1 \leq s \leq N^{2}\right]$ is the tri-block diagonal matrix with

$$
\begin{aligned}
& P_{(m-1) N+l,(m-1) N+l}=h^{2}\left[2 G_{l, m}^{(1)}+2 G_{l, m}^{(2)}-2 H_{x l, m}^{(1)}-2 I_{y l, m}^{(2)}-2 G_{l, m}^{(3)}+H_{l, m}^{(1)} H_{l, m}^{(3)}+I_{l, m}^{(2)} I_{l, m}^{(3)}\right] \\
&+ O\left(h^{4}\right) \quad[1 \leq l \leq N, 1 \leq m \leq N], \\
& P_{(m-1) N+l,(m-1) N+l \pm 1}= h\left[ \pm 2 H_{l, m}^{(1)} \pm H_{l, m}^{(2)} \mp H_{l, m}^{(3)}\right]+\frac{h^{2}}{2}\left[2 G_{l, m}^{(1)}+2 H_{x l, m}^{(1)}-H_{l, m}^{(1)} H_{l, m}^{(3)}\right] \\
&+O\left(h^{3}\right) \quad[1 \leq l \leq N-1,2 \leq l \leq N, 1 \leq m \leq N], \\
& P_{(m-1) N+l,(m-1 \pm 1) N+l}= h\left[ \pm 2 I_{l, m}^{(2)} \pm I_{l, m}^{(1)} \mp I_{l, m}^{(3)}\right]+\frac{h^{2}}{2}\left[2 G_{l, m}^{(2)}+2 I_{y l, m}^{(2)}-I_{l, m}^{(2)} I_{l, m}^{(3)}\right] \\
&+O\left(h^{3}\right) \quad[1 \leq l \leq N, 1 \leq m \leq N-1,2 \leq m \leq N],
\end{aligned}
$$




$$
\begin{aligned}
P_{(m-1) N+l, m N+l \pm 1}= & \frac{h}{2}\left[ \pm H_{l, m}^{(2)}+I_{l, m}^{(1)}\right]+\frac{h^{2}}{8}\left[ \pm 2 H_{y l, m}^{(2)} \pm 2 I_{x l, m}^{(1)} \mp I_{l, m}^{(1)} H_{l, m}^{(3)} \mp H_{l, m}^{(2)} I_{l, m}^{(3)}\right] \\
& +O\left(h^{3}\right) \quad[1 \leq l \leq N-1,2 \leq l \leq N, 1 \leq m \leq N-1], \\
P_{(m-1) N+l,(m-2) N+l \pm 1}= & \frac{h}{2}\left[ \pm H_{l, m}^{(2)}-I_{l, m}^{(1)}\right]+\frac{h^{2}}{8}\left[\mp 2 H_{y l, m}^{(2)} \mp 2 I_{x l, m}^{(2)} \pm I_{l, m}^{(1)} H_{l, m}^{(3)} \pm H_{l, m}^{(2)} I_{l, m}^{(3)}\right] \\
& +O\left(h^{3}\right) \quad[1 \leq l \leq N-1,2 \leq l \leq N, 2 \leq m \leq N] .
\end{aligned}
$$

Using relation (29), from equations (25) and (26), in the absence of round-off errors, we obtain the error equation

$$
(\mathbf{D}+\mathbf{P}) \mathbf{E}=\overline{\mathbf{T}}
$$

Let $\bar{R}=R \cup \partial R$ and

$$
G_{*}=\min _{(x, y) \in \bar{R}} \frac{\partial f}{\partial U} \quad \text { and } \quad G^{*}=\max _{(x, y) \in \bar{R}} \frac{\partial f}{\partial U}
$$

then

$$
0<G_{*} \leq G_{l \pm \frac{1}{2}, m}^{(1)}, G_{l, m \pm \frac{1}{2}}^{(2)}, G_{l, m}^{(3)} \leq G^{*}
$$

and for $Q=H$ and $I$, let

$$
0<\left|Q_{l \pm \frac{1}{2}, m}^{(1)}\right|,\left|Q_{l, m \pm \frac{1}{2}}^{(2)}\right|,\left|Q_{l, m}^{(3)}\right| \leq Q
$$

and

$$
\left|Q_{x l, m}^{(1)}\right| \leq Q^{(1)}, \quad\left|Q_{y l, m}^{(2)}\right| \leq Q^{(2)}
$$

for some positive constants $Q, Q^{(1)}$ and $Q^{(2)}$.

Now, it is easy to verify that for sufficiently small $h$,

$$
\begin{array}{ll}
\left|P_{(m-1) N+l,(m-1) N+l}\right|<20 & {[1 \leq l \leq N, 1 \leq m \leq N],} \\
\left|P_{(m-1) N+l,(m-1) N+l \pm 1}\right|<4 & {[1 \leq l \leq N-1,2 \leq l \leq N, 1 \leq m \leq N]} \\
\left|P_{(m-1) N+l,(m-1 \pm 1) N+l}\right|<4 & {[1 \leq l \leq N, 1 \leq m \leq N-1,2 \leq m \leq N]} \\
\left|P_{(m-1) N+l, m N+l \pm 1}\right|<1 & {[1 \leq l \leq N-1,2 \leq l \leq N, 1 \leq m \leq N-1]} \\
\left|P_{(m-1) N+l,(m-2) N+l \pm 1}\right|<1 & {[1 \leq l \leq N-1,2 \leq l \leq N, 2 \leq m \leq N] .}
\end{array}
$$

Further, the directed graph of $\mathbf{D}+\mathbf{P}$ shows that it is an irreducible matrix (see Figure 2). The arrows indicate the paths $i \rightarrow j$ for every nonzero entry $(\mathbf{D}+\mathbf{P})_{(i, j)}$ of the matrix $\mathbf{D}+\mathbf{P}$. For any ordered pair of nodes $i$ and $j$, there exists a direct path $\left(\overrightarrow{i, l_{1}}\right),\left(\overrightarrow{l_{1}}, l_{2}\right), \ldots,\left(\overrightarrow{l_{k}}, j\right)$ connecting $i$ to $j$. Hence, the graph is strongly connected. So, the matrix $\mathbf{D}+\mathbf{P}$ is irreducible (see Varga [21]). 


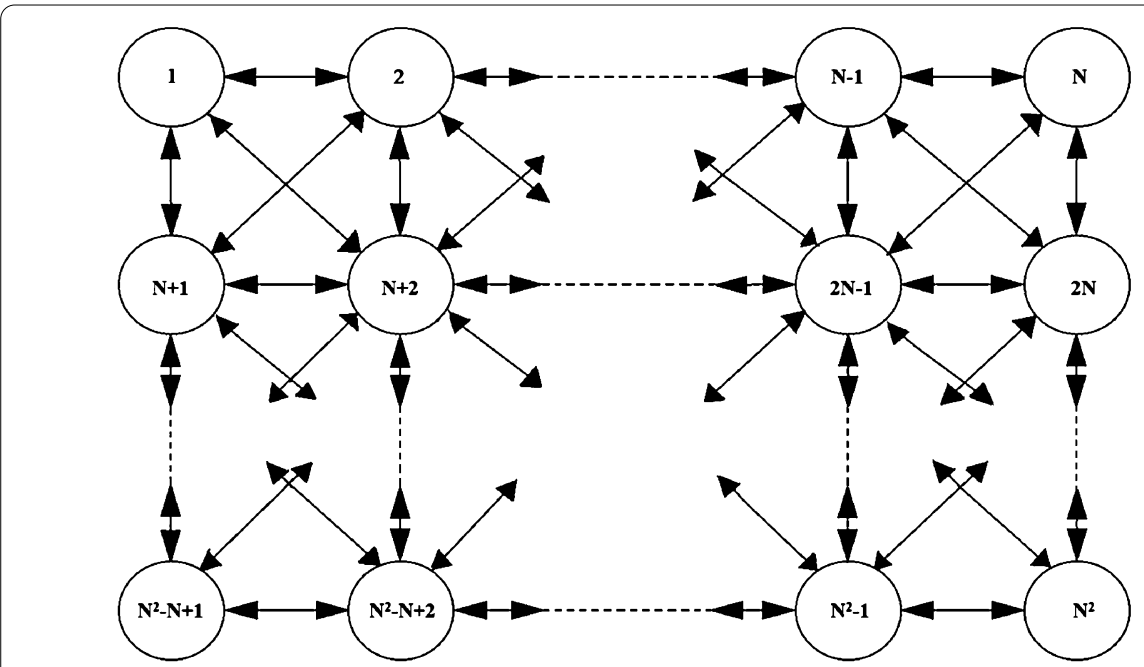

Figure 2 Directed graph of $\mathbf{D}+\mathbf{P}$.

Let $S_{k}$ denote the sum of the elements in the $k$ th row of $\mathbf{D}+\mathbf{P}$, then for $k=1$ and $N$, we have

$$
S_{k}=11(A+B) / 2+h^{2}\left(3 G_{k, 1}^{(1)}+3 G_{k, 1}^{(2)}-2 G_{k, 1}^{(3)}\right)+\frac{h}{8}\left(b_{k}+h c_{k}\right)+O\left(h^{3}\right),
$$

where

$$
\begin{aligned}
b_{k}= \pm 16 H_{k, 1}^{(1)} \pm 12 H_{k, 1}^{(2)}+16 I_{k, 1}^{(2)}+12 I_{k, 1}^{(1)} \mp 8 H_{k, 1}^{(3)}-8 I_{k, 1}^{(3)}, \\
c_{k}=4 H_{k, 1}^{(1)} H_{k, 1}^{(3)}+4 I_{k, 1}^{(2)} I_{k, 1}^{(3)}-8 H_{x k, 1}^{(1)}-8 I_{y k, 1}^{(2)} \pm 2 H_{y k, 1}^{(2)} \pm 2 I_{x k, 1}^{(1)} \mp I_{k, 1}^{(1)} H_{k, 1}^{(3)} \mp H_{k, 1}^{(2)} I_{k, 1}^{(3)}, \\
S_{(N-1) N+k=} 11(A+B) / 2+h^{2}\left[3 G_{k, N}^{(1)}+3 G_{k, N}^{(2)}-2 G_{k, N}^{(3)}\right] \\
+\frac{h}{8}\left[b_{(N-1) N+k}+h c_{(N-1) N+k}\right]+O\left(h^{3}\right),
\end{aligned}
$$

where

$$
\begin{aligned}
b_{(N-1) N+k}= & \pm 16 H_{k, N}^{(1)} \pm 12 H_{k, N}^{(2)}-16 I_{k, N}^{(2)}-12 I_{k, N}^{(1)} \mp 8 H_{k, N}^{(3)}+8 I_{k, N}^{(3)}, \\
c_{(N-1) N+k}= & 4 H_{k, N}^{(1)} H_{k, N}^{(3)}+4 I_{k, N}^{(2)} I_{k, N}^{(3)}-8 H_{x k, N}^{(1)}-8 I_{y k, N}^{(2)} \\
& \mp 2 H_{y k, N}^{(2)} \mp 2 I_{x k, N}^{(1)} \pm I_{k, N}^{(1)} H_{k, N}^{(3)} \pm H_{k, N}^{(2)} I_{k, N}^{(3)} .
\end{aligned}
$$

For $2 \leq q \leq N-1$,

$$
S_{(q-1) N+k}=6 A+h^{2}\left[3 G_{k, q}^{(1)}+4 G_{k, q}^{(2)}-2 G_{k, q}^{(3)}\right]+\frac{h}{2}\left[b_{(q-1) N+k}+h c_{(q-1) N+k}\right]+O\left(h^{3}\right),
$$

where

$$
\begin{aligned}
& b_{(q-1) N+k}= \pm 4 H_{k, q}^{(1)} \pm 4 H_{k, q}^{(2)} \mp 2 H_{k, q^{\prime}}^{(3)} \\
& c_{(q-1) N+k}=-2 H_{x k, q}^{(1)}+H_{k, q}^{(1)} H_{k, q^{\prime}}^{(3)} .
\end{aligned}
$$


For $2 \leq r \leq N-1$,

$$
S_{(k-1) N+r}=6 B+h^{2}\left[4 G_{r, k}^{(1)}+3 G_{r, k}^{(2)}-2 G_{r, k}^{(3)}\right]+\frac{h}{2}\left[b_{(k-1) N+r}+h c_{(k-1) N+r}\right]+O\left(h^{3}\right),
$$

where

$$
\begin{aligned}
& b_{(k-1) N+r}= \pm 4 I_{r, k}^{(1)} \pm 4 I_{r, k}^{(2)} \mp 2 I_{r, k}^{(3)}, \\
& c_{(k-1) N+r}=-2 I_{y r, k}^{(2)}+I_{r, k}^{(2)} I_{r, k}^{(3)} .
\end{aligned}
$$

And finally, for $q=2(1) N-1, r=2(1) N-1$,

$$
S_{(r-1) N+q}=h^{2}\left[4 G_{q, r}^{(1)}+4 G_{q, r}^{(2)}-2 G_{q, r}^{(3)}\right]+O\left(h^{4}\right) .
$$

With the help of equations (31.1)-(31.5), we get

$$
\begin{aligned}
& \left|b_{k}\right| \leq 36(H+I) \text {, } \\
& \left|c_{k}\right| \leq 4\left(H^{2}+I^{2}\right)+8\left(H^{(1)}+I^{(2)}\right)+2\left(H^{(2)}+I^{(1)}\right)+2 I H \\
& \text { for } k=1, N,(N-1) N+1 \text { and } N^{2} \text {, } \\
& \left|b_{k}\right| \leq 10 H \\
& \left|c_{k}\right| \leq 2 H^{(1)}+H^{2} \\
& \text { for } k=(q-1) N+1 \text { and } q N ; 2 \leq q \leq N-1, \\
& \left|b_{k}\right| \leq 10 I \\
& \left|c_{k}\right| \leq 2 I^{(2)}+I^{2} \\
& \text { for } k=r \text { and }(N-1) N+r ; 2 \leq r \leq N-1 \text {. }
\end{aligned}
$$

It follows that for sufficiently small $h$,

$$
\begin{aligned}
& S_{k}>6 h^{2} G_{*} \\
& \quad \text { for } k=1, N,(N-1) N+1 \text { and } N^{2}, \\
& S_{k}>7 h^{2} G_{*} \\
& \quad \text { for } k=(q-1) N+1 \text { and } q N ; 2 \leq q \leq N-1, \\
& S_{k}>7 h^{2} G_{*} \\
& \quad \text { for } k=r \text { and }(N-1) N+r ; 2 \leq r \leq N-1, \\
& S_{(r-1)(N-1)+q} \geq h^{2}\left(8 G_{*}-2 G^{*}\right)>0, \quad \text { assuming } G^{*}<4 G_{*} \\
& \quad \text { for } 2 \leq q \leq N-1 \text { and } 2 \leq r \leq N-1 .
\end{aligned}
$$

Thus, for sufficiently small $h, \mathbf{D}+\mathbf{P}$ is monotone. Hence $(\mathbf{D}+\mathbf{P})^{-1}$ exists and $(\mathbf{D}+\mathbf{P})^{-1}=$ $\mathbf{J}^{-1}>\mathbf{0}$ (see Henrici [22]), where

$$
\mathbf{J}=\left(J_{r, s}\right) \quad\left[1 \leq r \leq N^{2} \text { and } 1 \leq s \leq N^{2}\right]
$$


Since

$$
\sum_{r=1}^{N^{2}} J_{p, r} S_{r}=1, \quad 1 \leq p \leq N^{2}
$$

and $G_{*}>0$, from equations (32.1)-(32.4), with $1 \leq p \leq N^{2}$, it follows that

$$
\begin{aligned}
& J_{p, k} \leq \frac{1}{S_{k}}<\frac{1}{6 h^{2} G_{*}} \\
& {\left[k=1, N,(N-1) N+1 \text { and } N^{2}\right],} \\
& \sum_{q=2}^{N-1} J_{p, k} S_{k} \leq \frac{1}{\min _{2 \leq q \leq N-1} S_{k}}<\frac{1}{7 h^{2} G_{*}} \\
& {[k=(q-1) N+1 \text { and } q N, 2 \leq q \leq N-1],} \\
& \sum_{r=2}^{N-1} J_{p, k} \leq \frac{1}{\min _{2 \leq r \leq N-1} S_{k}}<\frac{1}{7 h^{2} G_{*}} \\
& {[k=r \text { and }(N-1) N+r, 2 \leq r \leq N-1],} \\
& \sum_{q=2}^{N-2} \sum_{r=2}^{N-2} J_{p, k} \leq \frac{1}{\min _{2 \leq q \leq N-2} S_{k}} \leq \frac{1}{h^{2}\left(8 G_{*}-2 G^{*}\right)} \\
& {[k=(r-1) N+q, 2 \leq q \leq N-1 \text { and } 2 \leq r \leq N-1] .}
\end{aligned}
$$

Equation (30) may be written as

$$
\|\mathbf{E}\| \leq\|\mathbf{J}\|\|\mathbf{T}\|
$$

where

$$
\begin{aligned}
\|\mathbf{J}\|= & \max _{1 \leq p \leq N^{2}}\left[\left(J_{p, 1}+\sum_{q=2}^{(N-1)} J_{p, q}+J_{p, N}\right)+\left(\sum_{q=2}^{N-1} J_{p,(q-1) N+1}+\sum_{q=2}^{N-1} \sum_{r=2}^{N-1} J_{p,(q-1) N+r}+\sum_{q=2}^{N-1} J_{p, q N}\right)\right. \\
& \left.+\left(J_{p,(N-1) N+1}+\sum_{q=2}^{N-1} J_{p,(N-1) N+q}+J_{p, N^{2}}\right)\right]
\end{aligned}
$$

Using equations (33.1)-(33.4) in equation (35), from (34) we obtain, for sufficiently small $h$,

$$
\|\mathbf{E}\| \leq O\left(h^{4}\right)
$$

This establishes the convergence of the fourth-order difference method (9) (with $n=1$ ) for the scalar elliptic equation (23).

\section{Stability analysis}

We consider the steady state two-dimensional convection-diffusion equation

$$
u_{x x}+u_{y y}=\beta u_{x}, \quad(x, y) \in R,
$$


where $\beta=(1 / \varepsilon)>0$ is a constant, with $\varepsilon$ (the perturbation parameter) being the ratio of convective velocity to the diffusion coefficient.

Applying the difference scheme (9) with $\bar{T}_{l, m}=0$ to the above equation and letting $\tau=$ $(\beta h / 2)>0$, which is called the cell Reynolds number, we obtain

$$
\begin{aligned}
(20 & \left.+4 \tau^{2}\right) u_{l, m} \\
= & (1+\tau) u_{l-1, m-1}+4 u_{l, m-1}+(1-\tau) u_{l+1, m-1}+2\left(5-\tau+\tau^{2}\right) u_{l-1, m} \\
& +2\left(-1+\tau+\tau^{2}\right) u_{l+1, m}+(1+\tau) u_{l-1, m+1}+4 u_{l, m+1}+(1-\tau) u_{l+1, m+1} .
\end{aligned}
$$

The above is a system of $N^{2}$ number of linear equations in $N^{2}$ number of unknowns, which may be expressed in the matrix form as $\mathbf{A u}=\mathbf{B}$, where

$$
\begin{aligned}
& \mathbf{A}=\left[\begin{array}{lll}
\mathbf{P} & \mathbf{Q} & \mathbf{P}
\end{array}\right]_{N^{2} \times N^{2}} \quad(\text { Tri-block-diagonal matrix), } \\
& \mathbf{P}=\left[\begin{array}{lll}
1+\tau & 4 & 1-\tau
\end{array}\right]_{N \times N} \quad(\text { Tri-diagonal matrix }), \\
& \mathbf{Q}=\left[\begin{array}{lll}
2\left(5-\tau+\tau^{2}\right) & -\left(20+4 \tau^{2}\right) & 2\left(-1+\tau+\tau^{2}\right)
\end{array}\right]_{N \times N} \quad \text { (Tri-diagonal matrix), } \\
& \mathbf{B}: N^{2} \times 1 \quad \text { matrix consisting of boundary values. }
\end{aligned}
$$

Now, applying the Jacobi iteration method to the above system of equations, we obtain

$$
\begin{aligned}
(20 & \left.+4 \tau^{2}\right) u_{l, m}^{(s+1)} \\
= & (1+\tau) u_{l-1, m-1}^{(s)}+4 u_{l, m-1}^{(s)}+(1-\tau) u_{l+1, m-1}^{(s)}+2\left(5-\tau+\tau^{2}\right) u_{l-1, m}^{(s)} \\
& +2\left(-1+\tau+\tau^{2}\right) u_{l+1, m}^{(s)}+(1+\tau) u_{l-1, m+1}^{(s)}+4 u_{l, m+1}^{(s)}+(1-\tau) u_{l+1, m+1}^{(s)},
\end{aligned}
$$

where $s=0,1,2, \ldots$

We examine the stability of (39) by assuming that an error $\varepsilon_{l, m}^{(s)}$ exists at each grid point $(l, m)$ at the $s$ th iteration. We analyze the behavior of the error $\varepsilon_{l, m}^{(s)}$ by assuming it to be of the form

$$
\varepsilon_{l, m}^{(s)}=\xi^{s} A^{l} B^{m} \sin \left(\frac{\pi a l}{N}\right) \sin \left(\frac{\pi b m}{N}\right), \quad 1 \leq a, b \leq N,
$$

where $A$ and $B$ are arbitrary constants and $\xi$ is the propagating factor which determines the rate of growth or decay of the errors. The necessary and sufficient condition for the iterative method to be stable is $|\xi|<1$.

Using (40) in (39), the propagating factor for the Jacobi iteration method is obtained as

$$
\begin{aligned}
\xi_{J} & =\frac{\left[(1-\tau) \cos \left(\frac{\pi b}{N}\right)-1+\tau+\tau^{2}\right]^{1 / 2}\left[(1+\tau) \cos \left(\frac{\pi b}{N}\right)+5-\tau+\tau^{2}\right]^{1 / 2} \cos \left(\frac{\pi a}{N}\right)+2 \cos \left(\frac{\pi b}{N}\right)}{5+\tau^{2}}, \\
1 & \leq a, b \leq N
\end{aligned}
$$

Thus, the Jacobi Iteration method is stable for those values of $\tau$ such that $\left|\xi_{J}\right|<1$.

Similarly, applying the Gauss-Siedal iteration method to (38) and assuming the error at each grid point $(l, m)$ at the $s$ th iteration to be of the form (40), the corresponding propa- 
gation factor $\xi_{G S}$ is given by the equation

$$
\begin{aligned}
\eta^{3} & -4\left[2 \psi+\phi^{2}\left(5-\tau+\tau^{2}\right)(1-\tau) \cos \left(\frac{\pi b}{N}\right)\right] \eta^{2} \\
& +4\left[4 \psi^{2}-\left(5-\tau+\tau^{2}\right)\left(-1+\tau+\tau^{2}\right) \phi^{2}-\left(1-\tau^{2}\right) \cos ^{2}\left(\frac{\pi b}{N}\right) \phi^{2}\right] \eta \\
& -4 \phi^{2}(1+\tau)\left(-1+\tau+\tau^{2}\right) \cos \left(\frac{\pi b}{N}\right)=0, \quad 1 \leq a, b \leq N
\end{aligned}
$$

where $\eta=\xi_{G S}^{1 / 2}, \phi=\frac{\cos \left(\frac{\pi a}{N}\right)}{10+2 \tau^{2}}$ and $\psi=\frac{\cos \left(\frac{\pi b}{N}\right)}{10+2 \tau^{2}}$.

Thus, the Gauss-Siedal iteration method is stable for those values of $\tau$ such that $\left|\xi_{G S}\right|<1$.

\section{Generalisation of the above methods}

We now extend our methods to the system of 2D quasi-linear elliptic PDEs of the form:

$$
a^{(i)} u_{x x}^{(i)}+b^{(i)} u_{y y}^{(i)}=f^{(i)}, \quad 1 \leq i \leq n
$$

for $(x, y) \in R$, with each $a^{(i)}=a^{(i)}\left(x, y, u^{(1)}, u^{(2)}, \ldots, u^{(n)}\right), b^{(i)}=b^{(i)}\left(x, y, u^{(1)}, u^{(2)}, \ldots, u^{(n)}\right)$ and $f^{(i)}=f^{(i)}\left(x, y, u^{(1)}, u^{(2)}, \ldots, u^{(n)}, u_{x}^{(1)}, u_{x}^{(2)}, \ldots, u_{x}^{(n)}, u_{y}^{(1)}, u_{y}^{(2)}, \ldots, u_{y}^{(n)}\right)$, subject to the Dirichlet boundary conditions given by

$$
u^{(i)}(x, y)=v^{(i)}(x, y)
$$

We assume $U_{l, m}^{(i)}$ and $u_{l, m}^{(i)}$ to be the exact and approximate values of $u^{(i)}\left(x_{l}, y_{m}\right)$ respectively. For each $i=1(1) n$, letting $f_{l, m}^{(i)}=f^{(i)}\left(x_{l}, y_{m}, U_{l, m}^{(1)}, U_{l, m}^{(2)}, \ldots, U_{l, m}^{(n)}, U_{x l, m}^{(1)}, U_{x l, m}^{(2)}, \ldots, U_{x l, m}^{(n)}\right.$, $\left.U_{y l, m}^{(1)}, U_{y l, m}^{(2)}, \ldots, U_{y l, m}^{(n)}\right)$, we set the following approximations:

$$
\begin{aligned}
& \bar{U}_{l \pm \frac{1}{2}, m}^{(i)}=\left(U_{l \pm 1, m}^{(i)}+U_{l, m}^{(i)}\right) / 2, \\
& \bar{U}_{l, m \pm \frac{1}{2}}^{(i)}=\left(U_{l, m \pm 1}^{(i)}+U_{l, m}^{(i)}\right) / 2, \\
& \bar{U}_{x l, m}^{(i)}=\left(U_{l+1, m}^{(i)}-U_{l-1, m}^{(i)}\right) /(2 h), \\
& \bar{U}_{x l \pm \frac{1}{2}, m}^{(i)}=\left( \pm U_{l \pm 1, m}^{(i)} \mp U_{l, m}^{(i)}\right) /(h), \\
& \bar{U}_{x l, m \pm \frac{1}{2}}^{(i)}=\left(U_{l+1, m \pm 1}^{(i)}-U_{l-1, m \pm 1}^{(i)}+U_{l+1, m}^{(i)}-U_{l-1, m}^{(i)}\right) /(4 h), \\
& \bar{U}_{y l, m}^{(i)}=\left(U_{l, m+1}^{(i)}-U_{l, m-1}^{(i)}\right) /(2 h), \\
& \bar{U}_{y l \pm \frac{1}{2}, m}^{(i)}=\left(U_{l \pm 1, m+1}^{(i)}-U_{l \pm 1, m-1}^{(i)}+U_{l, m+1}^{(i)}-U_{l, m-1}^{(i)}\right) /(4 h), \\
& \bar{U}_{y l, m \pm \frac{1}{2}}^{(i)}=\left( \pm U_{l, m \pm 1}^{(i)} \mp U_{l, m}^{(i)}\right) /(h), \\
& \bar{U}_{x x l, m}^{(i)}=\left(U_{l+1, m}^{(i)}-2 U_{l, m}^{(i)}+U_{l-1, m}^{(i)}\right) /\left(h^{2}\right), \\
& \bar{U}_{x x l, m \pm 1}^{(i)}=\left(U_{l+1, m \pm 1}^{(i)}-2 U_{l, m \pm 1}^{(i)}+U_{l-1, m \pm 1}^{(i)}\right) /\left(h^{2}\right), \\
& \bar{U}_{y y l, m}^{(i)}=\left(U_{l, m+1}^{(i)}-2 U_{l, m}^{(i)}+U_{l, m-1}^{(i)}\right) /\left(h^{2}\right), \\
& \bar{U}_{y y l \pm 1, m}^{(i)}=\left(U_{l \pm 1, m+1}^{(i)}-2 U_{l \pm 1, m}^{(i)}+U_{l \pm 1, m-1}^{(i)}\right) /\left(h^{2}\right) . \\
&
\end{aligned}
$$


Define

$$
\begin{aligned}
\bar{f}_{l \pm \frac{1}{2}, m}^{(i)}= & f^{(i)}\left(x_{l \pm \frac{1}{2}}, y_{m}, \bar{U}_{l \pm \frac{1}{2}, m}^{(1)}, \bar{U}_{l \pm \frac{1}{2}, m}^{(2)}, \ldots, \bar{U}_{l \pm \frac{1}{2}, m}^{(n)}, \bar{U}_{x l \pm \frac{1}{2}, m}^{(1)},\right. \\
& \bar{U}_{x l \pm \frac{1}{2}, m}^{(2)}, \ldots, \bar{U}_{x l \pm \frac{1}{2}, m}^{(n)}, \bar{U}_{y l \pm \frac{1}{2}, m}^{(1)}, \\
& \left.\bar{U}_{y l \pm \frac{1}{2}, m}^{(2)}, \ldots, \bar{U}_{y l \pm \frac{1}{2}, m}^{(n)}\right), \\
\bar{f}_{l, m \pm \frac{1}{2}}^{(i)}= & f^{(i)}\left(x_{l}, y_{m \pm \frac{1}{2}}, \bar{U}_{l, m \pm \frac{1}{2}}^{(1)}, \bar{U}_{l, m \pm \frac{1}{2}}^{(2)}, \ldots, \bar{U}_{l, m \pm \frac{1}{2}}^{(n)}, \bar{U}_{x l, m \pm \frac{1}{2}}^{(1)},\right. \\
& \bar{U}_{x l, m \pm \frac{1}{2}}^{(2)}, \ldots, \bar{U}_{x l, m \pm \frac{1}{2}}^{(n)}, \bar{U}_{y l, m \pm \frac{1}{2}}^{(1)}, \\
& \left.\bar{U}_{y l, m \pm \frac{1}{2}}^{(2)}, \ldots, \bar{U}_{y l, m \pm \frac{1}{2}}^{(n)}\right) .
\end{aligned}
$$

Further, let

$$
\begin{aligned}
\overline{\bar{U}}_{l, m}^{(i)}= & U_{l, m}^{(i)}+\frac{h^{2}}{16 a_{00}^{(i)}}\left(\bar{f}_{l+\frac{1}{2}, m}^{(i)}+\bar{f}_{l-\frac{1}{2}, m}^{(i)}\right)+\frac{h^{2}}{16 b_{00}^{(i)}}\left(\bar{f}_{l, m+\frac{1}{2}}^{(i)}+\bar{f}_{l, m-\frac{1}{2}}^{(i)}\right) \\
& +\frac{h^{2}}{8}\left(1-\frac{a_{00}^{(i)}}{b_{00}^{(i)}}\right) \bar{U}_{x x l, m}^{(i)}+\frac{h^{2}}{8}\left(1-\frac{b_{00}^{(i)}}{a_{00}^{(i)}}\right) \bar{U}_{y y l, m}^{(i)}, \\
\overline{\bar{U}}_{x l, m}^{(i)}= & \bar{U}_{x l, m}^{(i)}+\frac{h}{4 a_{00}^{(i)}}\left(\bar{f}_{l+\frac{1}{2}, m}^{(i)}-\bar{f}_{l-\frac{1}{2}, m}^{(i)}\right)+\frac{h}{8}\left(1-\frac{b_{00}^{(i)}}{a_{00}^{(i)}}\right)\left(\bar{U}_{y y l+1, m}^{(i)}-\bar{U}_{y y l-1, m}^{(i)}\right) \\
& -\frac{h^{2} a_{10}^{(i)} \bar{U}_{x x l, m}^{(i)}-\frac{h^{2} b_{10}^{(i)}}{4 a_{00}^{(i)}} \bar{U}_{y y l, m}^{(i)},}{4 a_{00}^{(i)}} \\
\overline{\bar{U}}_{y l, m}^{(i)}= & \bar{U}_{y l, m}^{(i)}+\frac{h}{4 b_{00}^{(i)}}\left(\bar{f}_{l, m+\frac{1}{2}}^{(i)}-\bar{f}_{l, m-\frac{1}{2}}^{(i)}\right)+\frac{h}{8}\left(1-\frac{a_{00}^{(i)}}{b_{00}^{(i)}}\right)\left(\bar{U}_{x x l, m+1}^{(i)}-\bar{U}_{x x l, m-1}^{(i)}\right) \\
& -\frac{h^{2} a_{01}^{(i)} \bar{U}_{x x l, m}^{(i)}-\frac{h^{2} b_{01}^{(i)}}{4 b_{00}^{(i)}} \bar{U}_{y y l, m}^{(i)}}{4 b_{00}^{(i)}}
\end{aligned}
$$

and finally, we define

$$
\begin{aligned}
\overline{\bar{f}}_{l, m}^{(i)}= & f^{(i)}\left(x_{l}, y_{m}, \overline{\bar{U}}_{l, m}^{(1)}, \overline{\bar{U}}_{l, m}^{(2)}, \ldots, \overline{\bar{U}}_{l, m}^{(n)}, \overline{\bar{U}}_{x l, m}^{(1)}, \overline{\bar{U}}_{x l, m}^{(2)}, \ldots, \overline{\bar{U}}_{x l, m}^{(n)},\right. \\
& \left.\overline{\bar{U}}_{y l, m}^{(1)}, \overline{\bar{U}}_{y l, m}^{(2)}, \ldots, \overline{\bar{U}}_{y l, m}^{(n)}\right) .
\end{aligned}
$$

Then, at each internal grid point $(l, m)$, the fourth-order off-step discretization to each differential equation of system (43) is given by

$$
\begin{aligned}
L\left[U^{(i)}\right] \equiv & {\left[I_{1}^{(i)} \delta_{x}^{2}+I_{2}^{(i)} \delta_{y}^{2}+I_{3}^{(i)}\left(2 \delta_{x}^{2} \mu_{y} \delta_{y}\right)+I_{4}^{(i)}\left(2 \delta_{y}^{2} \mu_{x} \delta_{x}\right)+I_{5}^{(i)}\left(\delta_{x}^{2} \delta_{y}^{2}\right)\right] U_{l, m}^{(i)} } \\
= & h^{2}\left[J_{1}^{(i)} \bar{f}_{l+\frac{1}{2}, m}^{(i)}+J_{2}^{(i)} \bar{f}_{l-\frac{1}{2}, m}^{(i)}+J_{3}^{(i)} \bar{f}_{l, m+\frac{1}{2}}^{(i)}+J_{4}^{(i)} \bar{f}_{l, m-\frac{1}{2}}^{(i)}-2 \overline{\bar{f}}_{l, m}^{(i)}\right] \\
& +\bar{T}_{l, m}^{(i)} ; \quad 1 \leq l, m \leq N
\end{aligned}
$$


for $\bar{T}_{l, m}^{(i)}=O\left(h^{6}\right)$, where we denote

$$
\begin{aligned}
K_{1}^{(i)}= & \left(a_{l+1, m}^{(i)}-a_{l-1, m}^{(i)}\right) / 2 h a_{00}^{(i)}, \quad K_{2}^{(i)}=\left(b_{l, m+1}^{(i)}-b_{l, m-1}^{(i)}\right) / 2 h b_{00}^{(i)}, \\
I_{1}^{(i)}= & 4 a_{00}^{(i)}+\frac{1}{2}\left[a_{l+1, m}^{(i)}+a_{l-1, m}^{(i)}+a_{l, m+1}^{(i)}+a_{l, m-1}^{(i)}\right] \\
& -\frac{\left(a_{l, m+1}^{(i)}-a_{l, m-1}^{(i)}\right)\left(b_{l, m+1}^{(i)}-b_{l, m-1}^{(i)}\right)}{4 b_{00}^{(i)}}-\frac{\left(a_{l+1, m}^{(i)}-a_{l-1, m}^{(i)}\right)^{2}}{4 a_{00}^{(i)}}+O\left(h^{4}\right), \\
I_{2}^{(i)}= & 4 b_{00}^{(i)}+\frac{1}{2}\left[b_{l+1, m}^{(i)}+b_{l-1, m}^{(i)}+b_{l, m+1}^{(i)}+b_{l, m-1}^{(i)}\right] \\
& -\frac{\left(a_{l+1, m}^{(i)}-a_{l-1, m}^{(i)}\right)\left(b_{l+1, m}^{(i)}-b_{l-1, m}^{(i)}\right)}{4 a_{00}^{(i)}}-\frac{\left(b_{l, m+1}^{(i)}-b_{l, m-1}^{(i)}\right)^{2}}{4 b_{00}^{(i)}}+O\left(h^{4}\right), \\
I_{3}^{(i)}= & \frac{\left(a_{l, m+1}^{(i)}-a_{l, m-1}^{(i)}\right)}{4}-\left(\frac{b_{l, m+1}^{(i)}-b_{l, m-1}^{(i)}}{4 b_{00}^{(i)}}\right) a_{00}^{(i)}+O\left(h^{3}\right), \\
I_{4}^{(i)}= & \frac{\left(b_{l+1, m}^{(i)}-b_{l-1, m}^{(i)}\right)}{4}-\left(\frac{a_{l+1, m}^{(i)}-a_{l-1, m}^{(i)}}{4 a_{00}^{(i)}}\right) b_{00}^{(i)}+O\left(h^{3}\right), \\
I_{5}^{(i)}= & \frac{1}{2}\left(a_{00}^{(i)}+b_{00}^{(i)}\right), \\
J_{1}^{(i)}= & 2-h K_{1}^{(i)}, \quad J_{2}^{(i)}=2+h K_{1}^{(i)}, \quad J_{3}^{(i)}=2-h K_{2}^{(i)}, \quad J_{4}^{(i)}=2+h K_{2}^{(i)} .
\end{aligned}
$$

After the fourth-order approximate solution to system (43) is determined upon solving the tri-block diagonal system of equations (49), it is easy to see that the fourth-order estimates of $\left(\partial u^{(i)} / \partial n\right)$ can be explicitly obtained using the following discretizations:

$$
\begin{aligned}
U_{x l, m}^{(i)}= & \frac{1}{2 h}\left(2 \mu_{x} \delta_{x}\right) U_{l, m}^{(i)}+\frac{1}{12 h a_{00}^{(i)}}\left(\left(a_{l+1, m}^{(i)}-a_{l-1, m}^{(i)}\right) \delta_{x}^{2}+\left(b_{l+1, m}^{(i)}-b_{l-1, m}^{(i)}\right) \delta_{y}^{2}\right) U_{l, m}^{(i)} \\
& +\frac{b_{00}^{(i)}}{12 h a_{00}^{(i)}}\left(2 \mu_{x} \delta_{x}\right) \delta_{y}^{2} U_{l, m}^{(i)} \\
& -\frac{h}{6 a_{00}^{(i)}}\left(\bar{f}_{l+\frac{1}{2}, m}^{(i)}-\bar{f}_{l-\frac{1}{2}, m}^{(i)}\right)+\bar{T}_{l, m}^{(i, x)}, \quad 1 \leq l, m \leq N, \\
U_{y l, m}^{(i)}= & \frac{1}{2 h}\left(2 \mu_{y} \delta_{y}\right) U_{l, m}^{(i)}+\frac{1}{12 h b_{00}^{(i)}}\left(\left(a_{l, m+1}^{(i)}-a_{l, m-1}^{(i)}\right) \delta_{x}^{2}+\left(b_{l, m+1}^{(i)}-b_{l, m-1}^{(i)}\right) \delta_{y}^{2}\right) U_{l, m}^{(i)} \\
& +\frac{a_{00}^{(i)}}{12 h b_{00}^{(i)}}\left(2 \mu_{y} \delta_{y}\right) \delta_{x}^{2} U_{l, m}^{(i)} \\
& -\frac{h}{6 b_{00}^{(i)}}\left(\bar{f}_{l, m+\frac{1}{2}}^{(i)}-\bar{f}_{l, m-\frac{1}{2}}^{(i)}\right)+\bar{T}_{l, m}^{(i, y)}, \quad 1 \leq l, m \leq N,
\end{aligned}
$$

where $\bar{T}_{l, m}^{(i, x)}$ and $\bar{T}_{l, m}^{(i, y)}$ are of $O\left(h^{4}\right)$.

\section{Computational implementation}

We implement the proposed method over three linear and seven nonlinear problems, including a quasi-linear problem, in Cartesian and polar coordinates. The exact solutions of the problems are given. The right-hand side functions and the Dirichlet boundary conditions are determined using the exact solutions. The system of linear difference equations is 
solved using the block iterative method and the system of nonlinear difference equations by the Newton-Raphson method (see Hageman and Young [23], Kelly [24] and Saad [25]). The iterations are terminated once the absolute error tolerance $\leq 10^{-12}$ has been reached. All the computations are done using MATLAB programming language.

Example 1 (Convection-diffusion equation)

$$
u_{x x}+u_{y y}=\beta u_{x}, \quad 0<x, y<1
$$

subject to the Dirichlet boundary conditions given by

$$
\begin{aligned}
& u(x, 0)=0, \\
& u(x, 1)=0 \quad \text { for } 0<x<1, \\
& u(0, y)=\sin \pi y, \\
& u(1, y)=2 \sin \pi y \quad \text { for } 0<y<1 .
\end{aligned}
$$

The solution $u$ to the above equation and its first-order derivatives $u_{x}$ and $u_{y}$ at the point $(0.5,0.5)$ are listed in Table 1 for $\beta=10$ and 50 . Figure 3 gives the plot of the numerical solution to Example 1.

Example 2 (Poisson's equation in $r-\theta$ plane)

$$
u_{r r}+\frac{\alpha}{r} u_{r}+\frac{1}{r^{2}} u_{\theta \theta}=G(r, \theta), \quad 0<r, \theta<1
$$

At $\alpha=1$ and 2, the above equation represents 2D Poisson's equation in cylindrical and spherical coordinates, respectively. The exact solution is $u=r^{2} \cos (\pi \theta)$.

The maximum absolute errors (MAE) in $u, u_{x}$ and $u_{y}$ are listed in Table 2 for $\alpha=1$ and 2 .

\begin{tabular}{|c|c|c|c|c|c|}
\hline \multirow{2}{*}{\multicolumn{2}{|c|}{$\boldsymbol{h}$}} & \multicolumn{2}{|c|}{ Proposed $O\left(h^{4}\right)$-methods } & \multicolumn{2}{|c|}{$O\left(h^{2}\right)$-Methods } \\
\hline & & $\beta=10$ & $\beta=\mathbf{5 0}$ & $\beta=10$ & $\beta=\mathbf{5 0}$ \\
\hline $1 / 8$ & $\begin{array}{l}u \\
u_{x} \\
u_{y}\end{array}$ & $\begin{array}{r}6.4316(-01) \\
-5.0091(-01) \\
1.0190(-12)\end{array}$ & $\begin{array}{r}9.0714(-01) \\
-1.8812(-01) \\
1.8459(-13)\end{array}$ & $\begin{array}{r}6.4284(-01) \\
-5.1892(-01) \\
-1.1529(-12)\end{array}$ & $\begin{array}{l}\text { Diverges } \\
\text { Diverges } \\
\text { Diverges }\end{array}$ \\
\hline $1 / 16$ & $\begin{array}{l}u \\
u_{x} \\
u_{y}\end{array}$ & $\begin{array}{r}6.4286(-01) \\
-5.0104(-01) \\
2.2231(-12)\end{array}$ & $\begin{array}{r}9.0637(-01) \\
-1.7821(-01) \\
4.5889(-15)\end{array}$ & $\begin{array}{r}6.4275(-01) \\
-5.0464(-01) \\
-3.1806(-12)\end{array}$ & $\begin{array}{r}9.0666(-01) \\
-1.7790(-01) \\
3.4461(-13)\end{array}$ \\
\hline $1 / 32$ & $\begin{array}{l}u \\
u_{x} \\
u_{y}\end{array}$ & $\begin{array}{r}6.4284(-01) \\
-5.0105(-01) \\
4.4296(-12)\end{array}$ & $\begin{array}{r}9.0637(-01) \\
-1.7821(-01) \\
6.8094(-15)\end{array}$ & $\begin{array}{r}6.4281(-01) \\
-5.0190(-01) \\
-6.4109(-12)\end{array}$ & $\begin{array}{r}9.0644(-01) \\
-1.7808(-01) \\
1.7764(-15)\end{array}$ \\
\hline $1 / 64$ & $\begin{array}{l}u \\
u_{x} \\
u_{y}\end{array}$ & $\begin{array}{r}6.4284(-01) \\
-5.0105(-01) \\
8.5484(-12)\end{array}$ & $\begin{array}{r}9.0637(-01) \\
-1.7821(-01) \\
4.7370(-15)\end{array}$ & $\begin{array}{r}6.4283(-01) \\
-5.0126(-01) \\
-1.2545(-11)\end{array}$ & $\begin{array}{r}9.0639(-01) \\
-1.7818(-01) \\
1.4211(-14)\end{array}$ \\
\hline
\end{tabular}
Figure 4 gives the plots of the exact and numerical solutions to Example 2.

Example 3 (Poisson's equation in $r-z$ plane)

$$
u_{r r}+\frac{\alpha}{r} u_{r}+u_{z z}=G(r, z), \quad 0<r, z<1
$$

Table 1 Example 1: convection diffusion equation $(51)$ at the point $(0.5,0.5)$ 

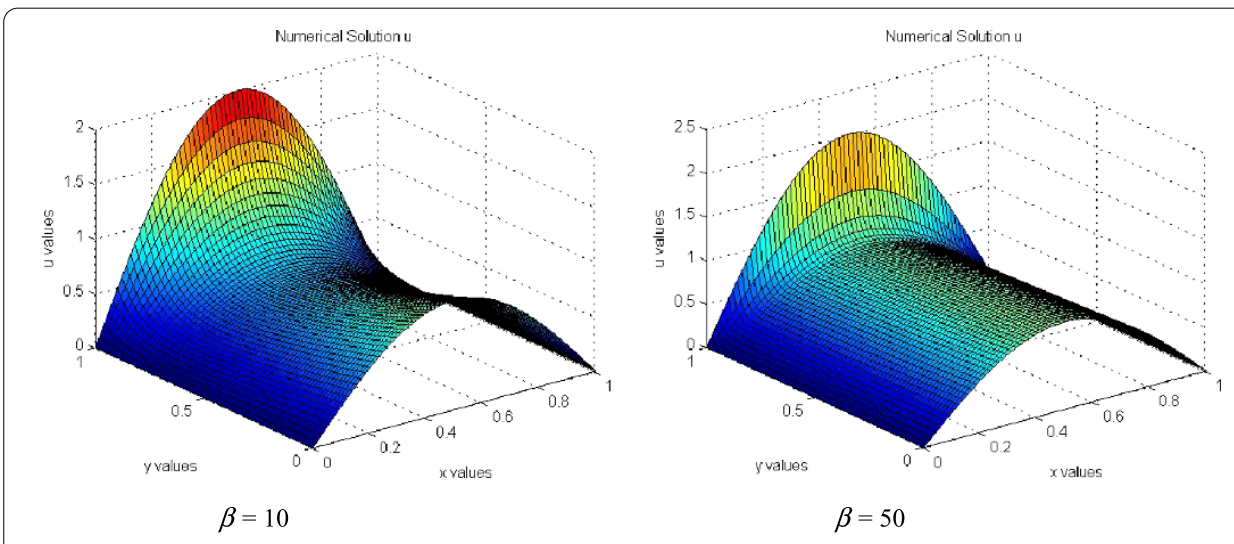

Figure 3 Numerical solutions of $2 \mathrm{D}$ convection diffusion equation at $(0.5,0.5)$.

Table 2 Example 2: Poisson's equations (52) in $r-\theta$ plane

\begin{tabular}{|c|c|c|c|c|c|}
\hline \multirow[t]{2}{*}{$\boldsymbol{h}$} & & \multicolumn{2}{|c|}{ Proposed $O\left(h^{4}\right)$-methods } & \multicolumn{2}{|c|}{$\begin{array}{l}O\left(h^{4}\right)-\text { Methods discussed } \\
\text { in }[16,17]\end{array}$} \\
\hline & & $\alpha=1$ & $\alpha=2$ & $\alpha=1$ & $\alpha=2$ \\
\hline \multirow[t]{3}{*}{$1 / 8$} & u & 2.3294(-06) & $4.6091(-06)$ & $4.2944(-06)$ & $6.8672(-06)$ \\
\hline & $u_{r}$ & $8.1179(-06)$ & $1.6529(-05)$ & $8.8246(-06)$ & $4.2834(-05)$ \\
\hline & $u_{\theta}$ & $3.9153(-04)$ & $6.2317(-04)$ & $4.4823(-04)$ & $8.6298(-04)$ \\
\hline \multirow[t]{3}{*}{$1 / 16$} & $u$ & $1.4731(-07)$ & $2.9153(-07)$ & $2.7278(-07)$ & $4.4629(-06)$ \\
\hline & $u_{r}$ & $9.7119(-07)$ & $1.8818(-06)$ & $8.1105(-07)$ & $3.0421(-06)$ \\
\hline & $u_{\theta}$ & $2.8537(-05)$ & $4.5514(-05)$ & $3.2188(-05)$ & $6.3244(-05)$ \\
\hline \multirow[t]{3}{*}{$1 / 32$} & $u$ & $9.2898(-09)$ & $1.8373(-08)$ & $1.8642(-08)$ & $3.2187(-07)$ \\
\hline & $u_{r}$ & 7.9264(-08) & $1.5078(-07)$ & $7.0243(-08)$ & $2.2424(-07)$ \\
\hline & $u_{\theta}$ & $1.9185(-06)$ & $3.0633(-06)$ & $2.3156(-06)$ & $4.4341(-06)$ \\
\hline \multirow[t]{3}{*}{$1 / 64$} & u & $5.8207(-10)$ & $1.1480(-09)$ & $1.1022(-09)$ & $1.9068(-08)$ \\
\hline & $u_{r}$ & $5.5941(-09)$ & $1.0553(-08)$ & $5.8124(-09)$ & $1.4172(-08)$ \\
\hline & $u_{\theta}$ & $1.2425(-07)$ & $1.9850(-07)$ & $1.5510(-07)$ & $2.7012(-07)$ \\
\hline
\end{tabular}

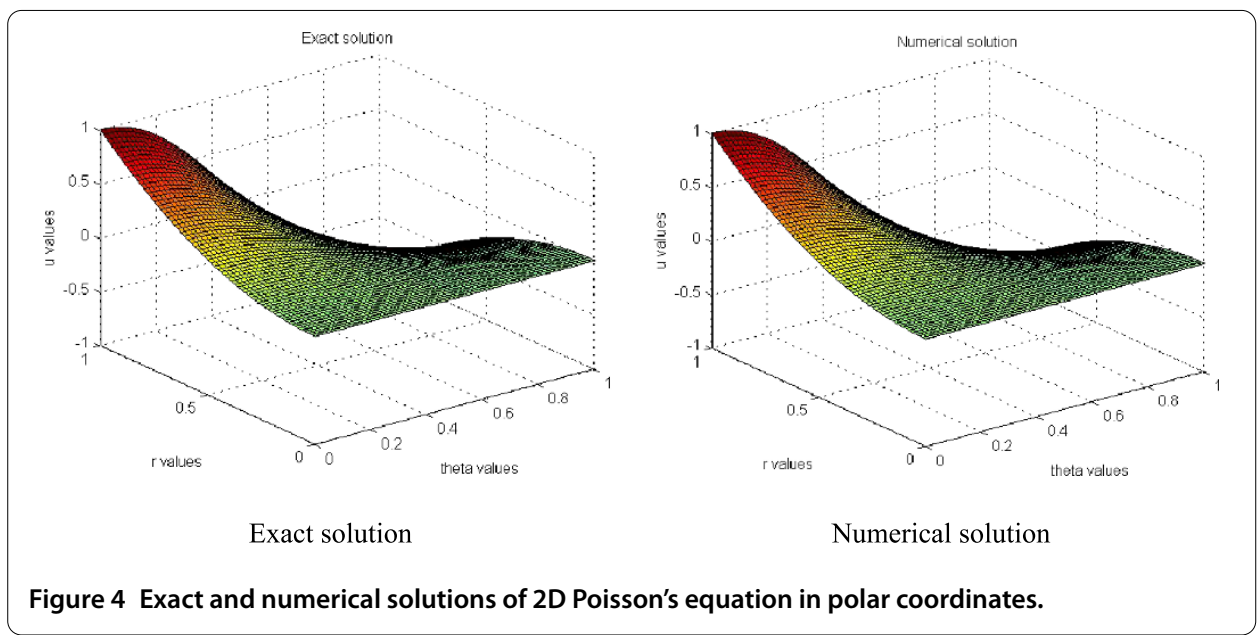


Table 3 Example 3: Poisson's equations (53) in $r$-z plane

\begin{tabular}{|c|c|c|c|c|c|}
\hline \multirow[t]{2}{*}{$\boldsymbol{h}$} & & \multicolumn{2}{|c|}{ Proposed $O\left(h^{4}\right)$-methods } & \multicolumn{2}{|c|}{$\begin{array}{l}O\left(h^{4}\right) \text {-Methods discussed } \\
\text { in }[16,17]\end{array}$} \\
\hline & & $\alpha=1$ & $\alpha=2$ & $\alpha=1$ & $\alpha=2$ \\
\hline $1 / 8$ & $\begin{array}{l}u \\
u_{r} \\
u_{z}\end{array}$ & $\begin{array}{l}1.6604(-06) \\
1.0721(-05) \\
2.3486(-05)\end{array}$ & $\begin{array}{l}2.8030(-06) \\
1.0390(-05) \\
3.6014(-05)\end{array}$ & $\begin{array}{l}2.8604(-06) \\
2.1444(-05) \\
3.6218(-05)\end{array}$ & $\begin{array}{l}4.2166(-06) \\
1.9833(-05) \\
4.8412(-05)\end{array}$ \\
\hline $1 / 16$ & $\begin{array}{l}u \\
u_{r} \\
u_{z}\end{array}$ & $\begin{array}{l}1.0530(-07) \\
8.1375(-07) \\
1.6895(-06)\end{array}$ & $\begin{array}{l}1.7649(-07) \\
8.1496(-07) \\
2.5690(-06)\end{array}$ & $\begin{array}{l}1.9884(-07) \\
1.1721(-06) \\
2.7520(-06)\end{array}$ & $\begin{array}{l}2.6261(-07) \\
1.0104(-06) \\
3.8224(-06)\end{array}$ \\
\hline $1 / 32$ & $\begin{array}{l}u \\
u_{r} \\
u_{z}\end{array}$ & $\begin{array}{l}6.6915(-09) \\
5.6905(-08) \\
1.1430(-07)\end{array}$ & $\begin{array}{l}1.1082(-08) \\
5.8312(-08) \\
1.7298(-07)\end{array}$ & $\begin{array}{l}1.1645(-08) \\
8.2169(-07) \\
1.6644(-07)\end{array}$ & $\begin{array}{l}1.6224(-08) \\
7.6186(-08) \\
2.3242(-07)\end{array}$ \\
\hline $1 / 64$ & $\begin{array}{l}u \\
u_{r} \\
u_{z}\end{array}$ & $\begin{array}{l}4.2259(-10) \\
4.5970(-09) \\
7.4703(-09)\end{array}$ & $\begin{array}{l}6.9292(-10) \\
7.3664(-09) \\
1.1278(-08)\end{array}$ & $\begin{array}{l}7.0120(-10) \\
5.0210(-08) \\
8.9744(-09)\end{array}$ & $\begin{array}{l}8.8844(-10) \\
4.5458(-09) \\
1.4242(-08)\end{array}$ \\
\hline
\end{tabular}

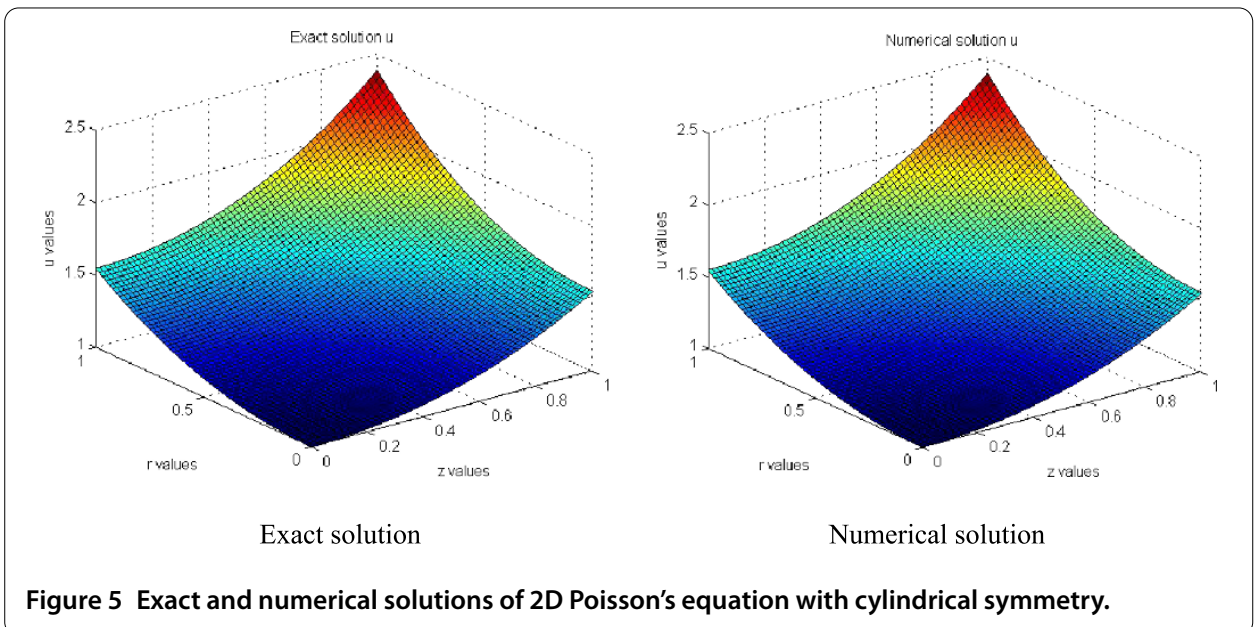

At $\alpha=1$, the above represents the two-dimensional Poisson's equation in cylindrical polar coordinates in $r-z$ plane. The exact solution is $u=\cosh r \cosh z$.

The MAE in $u, u_{x}$ and $u_{y}$ are listed in Table 3 for $\alpha=1$ and 2. Figure 5 gives the plots of the exact and numerical solutions to Example 3.

Example 4 (Burger's equation)

$$
\varepsilon\left(u_{x x}+u_{y y}\right)=u\left(u_{x}+u_{y}\right)+g(x, y), \quad 0<x, y<1 .
$$

The exact solution is $u=e^{x} \sin \left(\frac{\pi y}{2}\right)$. The MAE in $u, u_{x}$ and $u_{y}$ are listed in Table 4 for $\varepsilon=0.1,0.01$ and 0.001 .

Example 5 (Nonlinear elliptic equation)

$$
\left(1+x^{2}\right) u_{x x}+\left(1+y^{2}\right) u_{y y}=\alpha u\left(u_{x}+u_{y}\right)+f(x, y), \quad 0<x, y<1
$$

with the exact solution $u=e^{x} \sin (\pi y)$. The MAE for $u, u_{x}$ and $u_{y}$ are given in Table 5 for various values of $\alpha$. 
Table 4 Example 4: Burger's equation (54)

\begin{tabular}{|c|c|c|c|c|c|c|c|}
\hline \multirow{2}{*}{\multicolumn{2}{|c|}{$h$}} & \multicolumn{3}{|c|}{ Proposed $O\left(h^{4}\right)$-methods } & \multicolumn{3}{|c|}{$O\left(h^{4}\right)$-Methods discussed in $[16,17]$} \\
\hline & & $\varepsilon=0.1$ & $\varepsilon=0.01$ & $\varepsilon=0.001$ & $\varepsilon=0.1$ & $\varepsilon=0.01$ & $\varepsilon=0.001$ \\
\hline \multirow[t]{3}{*}{$1 / 16$} & $u$ & $2.0616(-05)$ & $2.4785(-04)$ & $2.5130(-03)$ & $4.4185(-05)$ & $4.8266(-04)$ & $3.9282(-03)$ \\
\hline & $u_{x}$ & $1.2837(-04)$ & $2.5155(-03)$ & $1.5332(-02)$ & $2.5208(-04)$ & $2.8427(-03)$ & $2.5834(-02)$ \\
\hline & $u_{y}$ & $1.6447(-04)$ & $1.3841(-03)$ & $1.5891(-02)$ & $3.0162(-04)$ & $2.7129(-03)$ & $2.4345(-02)$ \\
\hline \multirow[t]{3}{*}{$1 / 32$} & $u$ & $1.2695(-06)$ & $1.7462(-05)$ & $1.4391(-04)$ & $2.9287(-06)$ & $3.4324(-05)$ & $2.8411(-04)$ \\
\hline & $u_{x}$ & $1.6905(-05)$ & $2.0866(-04)$ & $1.6390(-03)$ & $1.9812(-05)$ & $2.2186(-04)$ & $2.6928(-03)$ \\
\hline & $u_{y}$ & $1.1825(-05)$ & $8.9167(-05)$ & $1.0726(-03)$ & $2.1441(-05)$ & $2.0412(-04)$ & $1.8184(-03)$ \\
\hline \multirow[t]{3}{*}{$1 / 64$} & $u$ & $7.9012(-08)$ & $1.1296(-06)$ & $1.0375(-05)$ & $1.8119(-07)$ & $2.1204(-06)$ & $2.1686(-05)$ \\
\hline & $u_{x}$ & $1.5530(-06)$ & $1.4118(-05)$ & $2.4812(-04)$ & $1.2124(-06)$ & $1.3016(-05)$ & $2.8928(-04)$ \\
\hline & $u_{y}$ & $8.0908(-07)$ & $6.2655(-06)$ & $5.4500(-05)$ & $1.2888(-06)$ & $1.2162(-05)$ & $1.1421(-04)$ \\
\hline
\end{tabular}

Table 5 Example 5: nonlinear equation (55)

\begin{tabular}{|c|c|c|c|c|c|c|c|}
\hline \multirow{2}{*}{\multicolumn{2}{|c|}{$\boldsymbol{h}$}} & \multicolumn{3}{|c|}{ Proposed $\boldsymbol{O}\left(h^{4}\right)$-methods } & \multicolumn{3}{|c|}{$O\left(h^{4}\right)$-Methods discussed in $[16,17]$} \\
\hline & & $\alpha=1$ & $\alpha=10$ & $\alpha=25$ & $\alpha=1$ & $\alpha=10$ & $\alpha=25$ \\
\hline \multirow[t]{3}{*}{$1 / 16$} & $u$ & $6.6498(-06)$ & $1.4642(-04)$ & $4.8477(-04)$ & $7.6488(-06)$ & $2.4122(-04)$ & $5.6678(-04)$ \\
\hline & $u_{x}$ & $9.4134(-05)$ & $1.3702(-03)$ & $3.1661(-03)$ & $1.1012(-04)$ & $2.3816(-03)$ & $4.0465(-03)$ \\
\hline & $u_{y}$ & $3.0028(-04)$ & $2.5306(-03)$ & $5.9721(-03)$ & $3.8149(-04)$ & $3.6296(-03)$ & $6.8764(-03)$ \\
\hline \multirow[t]{3}{*}{$1 / 32$} & $u$ & $4.1944(-07)$ & $9.1279(-06)$ & $2.9675(-05)$ & $4.8894(-07)$ & $1.5158(-03)$ & $3.6654(-05)$ \\
\hline & $u_{x}$ & $6.6876(-06)$ & $1.3007(-04)$ & $4.3732(-04)$ & $7.2724(-06)$ & $2.2244(-04)$ & $3.8975(-04)$ \\
\hline & $u_{y}$ & $2.0286(-05)$ & $1.7751(-04)$ & $4.1266(-04)$ & $2.6261(-05)$ & $2.4890(-04)$ & $4.8243(-04)$ \\
\hline \multirow[t]{3}{*}{$1 / 64$} & $u$ & $2.6367(-08)$ & $5.6914(-07)$ & $1.8430(-06)$ & $3.0189(-08)$ & $9.2284(-07)$ & $2.2056(-06)$ \\
\hline & $u_{x}$ & $4.4521(-07)$ & $1.0153(-05)$ & $4.3173(-05)$ & $4.8484(-07)$ & $1.8446(-05)$ & $3.6698(-05)$ \\
\hline & $u_{y}$ & $1.3205(-06)$ & $1.1963(-05)$ & $2.8454(-05)$ & $1.6185(-06)$ & $1.5177(-05)$ & $3.0125(-05)$ \\
\hline
\end{tabular}

Table 6 Example 6: quasi-linear equation (56)

\begin{tabular}{|c|c|c|c|c|c|c|c|}
\hline \multirow[t]{2}{*}{$\boldsymbol{h}$} & & \multicolumn{3}{|c|}{ Proposed $O\left(h^{4}\right)$-methods } & \multicolumn{3}{|c|}{$O\left(h^{4}\right)$-Methods discussed in $[16,17]$} \\
\hline & & $\alpha=1$ & $\alpha=5$ & $\alpha=10$ & $\alpha=1$ & $\alpha=5$ & $\alpha=10$ \\
\hline \multirow[t]{3}{*}{$1 / 16$} & $u$ & $2.5631(-05)$ & $3.8351(-05)$ & $3.0062(-04)$ & $4.5664(-05)$ & $5.8820(-05)$ & $4.9462(-04)$ \\
\hline & $u_{x}$ & $8.7514(-04)$ & $7.1537(-04)$ & $2.0151(-03)$ & $1.1016(-03)$ & $9.1939(-04)$ & $4.0466(-03)$ \\
\hline & $u_{y}$ & $2.7052(-03)$ & $2.0435(-03)$ & $1.5589(-03)$ & $4.8022(-03)$ & $3.9944(-03)$ & $3.6699(-03)$ \\
\hline \multirow[t]{3}{*}{$1 / 32$} & $u$ & $1.7057(-06)$ & $2.2772(-06)$ & $1.8240(-05)$ & $3.3186(-06)$ & $4.6368(-06)$ & $3.6864(-05)$ \\
\hline & $u_{x}$ & $6.4279(-05)$ & $5.7402(-05)$ & $1.7046(-04)$ & $7.6298(-05)$ & $7.8946(-05)$ & $2.7284(-04)$ \\
\hline & $u_{y}$ & $1.9020(-04)$ & $1.3883(-04)$ & $1.0118(-04)$ & $3.5122(-04)$ & $2.6674(-04)$ & $2.4468(-04)$ \\
\hline \multirow[t]{3}{*}{$1 / 64$} & $u$ & $1.0974(-07)$ & $1.4068(-07)$ & $1.1322(-06)$ & $1.9961(-07)$ & $2.8254(-07)$ & $2.2892(-06)$ \\
\hline & $u_{x}$ & $4.4614(-06)$ & $3.9908(-06)$ & $1.2621(-05)$ & $4.4324(-06)$ & $4.5540(-06)$ & $1.6884(-05)$ \\
\hline & $u_{y}$ & $1.2825(-05)$ & $9.0687(-06)$ & $6.3754(-06)$ & $2.3255(-05)$ & $1.6243(-05)$ & $1.4882(-05)$ \\
\hline
\end{tabular}

Example 6 (Quasi-linear elliptic equation)

$$
u_{x x}+\left(1+u^{2}\right) u_{y y}=\alpha u\left(u_{x}+u_{y}\right)+f(x, y), \quad 0<x, y<1 .
$$

The exact solution is $u=e^{x} \cos (\pi y)$. The MAE in $u, u_{x}$ and $u_{y}$ are tabulated in Table 6 .

Example 7 (2D steady-state Navier Stokes' model equations in Cartesian coordinates)

$$
\begin{array}{ll}
\frac{1}{R_{e}}\left(u_{x x}+u_{y y}\right)=u u_{x}+v u_{y}+f(x, y), & 0<x, y<1, \\
\frac{1}{R_{e}}\left(v_{x x}+v_{y y}\right)=u v_{x}+v v_{y}+g(x, y), & 0<x, y<1,
\end{array}
$$


Table 7 Example 7: Navier Stokes' model equations (57.1)-(57.2) in Cartesian coordinates

\begin{tabular}{|c|c|c|c|c|c|c|c|}
\hline \multirow[t]{2}{*}{$\boldsymbol{h}$} & & \multicolumn{3}{|c|}{ Proposed $O\left(h^{4}\right)$-methods } & \multicolumn{3}{|c|}{$O\left(h^{4}\right)$-Methods discussed in $[16,17]$} \\
\hline & & $R_{e}=10$ & $R_{e}=10^{2}$ & $R_{e}=10^{3}$ & $R_{e}=10$ & $R_{e}=10^{2}$ & $R_{e}=10^{3}$ \\
\hline \multirow[t]{6}{*}{$1 / 16$} & u & $3.8170(-05)$ & $7.9117(-04)$ & $1.1370(-02)$ & $4.2172(-05)$ & $8.1235(-04)$ & $1.4212(-02)$ \\
\hline & $v$ & $2.0205(-05)$ & $8.1179(-04)$ & $1.6068(-02)$ & $2.3785(-05)$ & $8.4455(-04)$ & $1.9872(-02)$ \\
\hline & $u_{x}$ & $9.4014(-04)$ & $9.2254(-03)$ & $4.4590(-01)$ & $9.5679(-04)$ & $9.6926(-03)$ & $4.9810(-01)$ \\
\hline & $v_{x}$ & $3.5771(-04)$ & $4.5339(-03)$ & $1.1926(-01)$ & $3.8239(-04)$ & $4.9830(-03)$ & $1.5879(-01)$ \\
\hline & $u_{y}$ & $3.8631(-04)$ & $4.4359(-03)$ & $1.3652(-01)$ & $4.1432(-04)$ & 4.8792(-03) & $1.7652(-01)$ \\
\hline & $v_{y}$ & $1.0075(-03)$ & $2.4480(-02)$ & $7.1490(-01)$ & $1.4087(-03)$ & $2.8880(-02)$ & $7.5451(-01)$ \\
\hline \multirow[t]{6}{*}{$1 / 32$} & $u$ & $2.4148(-06)$ & $4.4070(-05)$ & $6.8308(-04)$ & $2.7868(-06)$ & $4.7602(-05)$ & $7.6880(-04)$ \\
\hline & $v$ & $1.2680(-06)$ & 4.6982(-05) & $1.9517(-03)$ & $1.5742(-06)$ & 4.9852(-05) & $1.9702(-03)$ \\
\hline & $u_{x}$ & $6.2750(-05)$ & $8.3740(-04)$ & $1.5182(-02)$ & $6.4690(-05)$ & $8.6220(-04)$ & $1.8886(-02)$ \\
\hline & $v_{x}$ & $2.5272(-05)$ & $2.9250(-04)$ & $5.9531(-03)$ & $2.7160(-05)$ & $3.2280(-04)$ & $6.6122(-03)$ \\
\hline & $u_{y}$ & $2.4588(-05)$ & $3.9206(-04)$ & $1.5925(-02)$ & $2.7893(-05)$ & $4.2185(-04)$ & $1.6120(-02)$ \\
\hline & $v_{y}$ & $6.3855(-05)$ & $1.5382(-03)$ & $9.7499(-02)$ & $6.7984(-05)$ & $1.8790(-03)$ & $9.8242(-02)$ \\
\hline \multirow[t]{6}{*}{$1 / 64$} & u & $1.5149(-07)$ & $2.6562(-06)$ & $5.3901(-05)$ & $1.7044(-07)$ & $2.9885(-06)$ & $5.8280(-05)$ \\
\hline & $v$ & 7.9504(-08) & $3.0175(-06)$ & $1.0199(-04)$ & $8.4324(-08)$ & $3.3046(-06)$ & $1.2692(-04)$ \\
\hline & $u_{x}$ & $4.0049(-06)$ & $6.1189(-05)$ & $1.8824(-03)$ & $4.0049(-06)$ & $6.2772(-05)$ & $2.1982(-03)$ \\
\hline & $\hat{v_{x}}$ & $1.6940(-06)$ & $4.2246(-05)$ & $4.0036(-04)$ & $1.6944(-06)$ & $4.4468(-05)$ & $4.3322(-04)$ \\
\hline & $u_{y}$ & $1.5415(-06)$ & $3.1024(-05)$ & $1.1633(-03)$ & $1.5416(-06)$ & $3.3386(-05)$ & $1.2284(-03)$ \\
\hline & $v_{y}$ & $4.0005(-06)$ & $9.5734(-05)$ & $6.4802(-03)$ & $4.0003(-06)$ & $1.1248(-04)$ & $6.5748(-03)$ \\
\hline
\end{tabular}

where $R_{e}>0$ is a constant called the Reynolds number. The exact solution is $u=$ $\sin (\pi x) \sin (\pi y), v=\cos (\pi x) \cos (\pi y)$. The MAE in $u, v, u_{x}, v_{x}, u_{y}$ and $v_{y}$ are tabulated in Table 7 for $R_{e}=10,10^{2}$ and $10^{3}$. Figure 6 gives the plots of the exact and numerical solutions.

Example 8 (2D steady-state Navier Stokes' model equations in polar coordinates)

(a) In spherical polar coordinates in $r-\theta$ plane:

$$
\begin{aligned}
& \frac{1}{R_{e}}\left(u_{r r}+\frac{1}{r^{2}} u_{\theta \theta}+\frac{2}{r} u_{r}+\frac{\cot \theta}{r^{2}} u_{\theta}-\frac{2}{r^{2}} v_{\theta}-\frac{2}{r^{2}} u-\frac{2 \cot \theta}{r^{2}} v\right) \\
& \quad=u u_{r}+\frac{1}{r} v u_{\theta}-\frac{1}{r} v^{2}+H(r, \theta), \quad 0<r, \theta<1, \\
& \frac{1}{R_{e}}\left(v_{r r}+\frac{1}{r^{2}} v_{\theta \theta}+\frac{2}{r} v_{r}+\frac{\cot \theta}{r^{2}} v_{\theta}+\frac{2}{r^{2}} u_{\theta}-\frac{\cos e c^{2} \theta}{r^{2}} v\right) \\
& \quad=u v_{r}+\frac{1}{r} v v_{\theta}+\frac{1}{r} u v+I(r, \theta), \quad 0<r, \theta<1 .
\end{aligned}
$$

The exact solution is given by $u=2 r^{3} \cos \theta, v=-5 r^{3} \sin \theta$.

(b) In cylindrical polar coordinates in $r-\theta$ plane:

$$
\begin{aligned}
& \frac{1}{R_{e}}\left(u_{r r}+\frac{1}{r^{2}} u_{\theta \theta}+\frac{1}{r} u_{r}-\frac{2}{r^{2}} v_{\theta}-\frac{1}{r^{2}} u\right) \\
& \quad=u u_{r}+\frac{1}{r} v u_{\theta}-\frac{1}{r} v^{2}+H(r, \theta), \quad 0<r, \theta<1, \\
& \frac{1}{R_{e}}\left(v_{r r}+\frac{1}{r^{2}} v_{\theta \theta}+\frac{1}{r} v_{r}+\frac{2}{r^{2}} u_{\theta}-\frac{1}{r^{2}} v\right) \\
& \quad=u v_{r}+\frac{1}{r} v v_{\theta}+\frac{1}{r} u v+I(r, \theta), \quad 0<r, \theta<1 .
\end{aligned}
$$




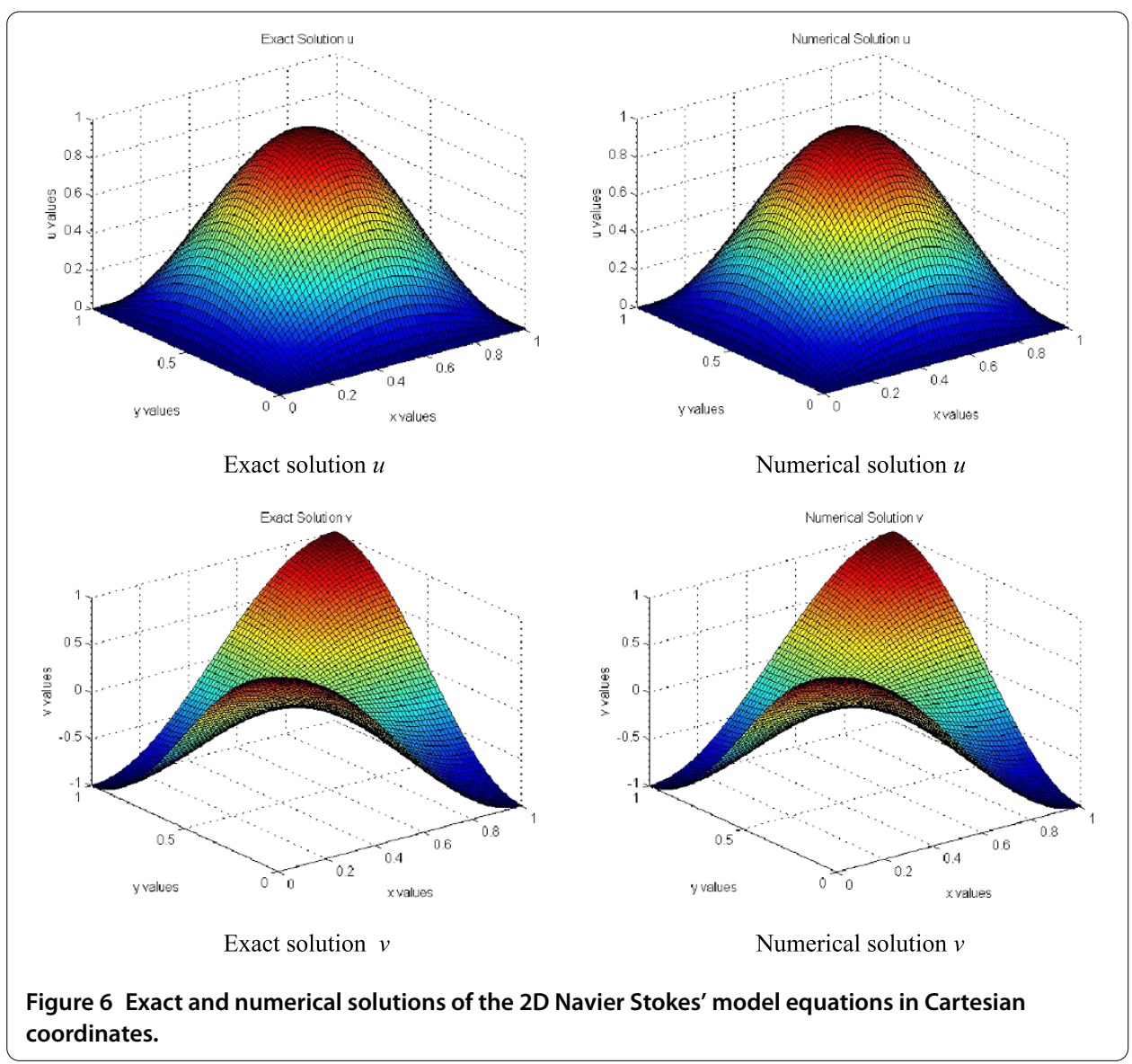

The exact solution is given by $u=r^{3} \sin \theta, v=4 r^{3} \cos \theta$.

(c) In cylindrical polar coordinates in $r-z$ plane:

$$
\begin{aligned}
& \frac{1}{R_{e}}\left(u_{r r}+\frac{1}{r} u_{r}+u_{z z}-\frac{1}{r^{2}} u\right)=u u_{r}+v u_{z}+H(r, z), \quad 0<r, z<1, \\
& \frac{1}{R_{e}}\left(v_{r r}+\frac{1}{r} v_{r}+v_{z z}\right)=u v_{r}+v v_{z}+I(r, z), \quad 0<r, z<1 .
\end{aligned}
$$

The exact solution is given by $u=r^{3} \sinh z, v=-4 r^{2} \cosh z$.

Here $R_{e}>0$ is called the Reynolds number. The MAE for $u, v$ and their first order normal derivatives are tabulated in Tables 8-10 for various values $R_{e}$. Figures 7, 8, 9 give a comparison of the plots of the exact and the numerical solutions to Example 8(a), (b) and (c) respectively.

\section{Concluding remarks}

The existing fourth-order nine-point difference methods of [16] for the numerical solution of the system of second-order quasi-linear 2D elliptic equations (43) require a special treatment to handle the numerical scheme at singular points. This is because of the appearance of terms, for instance, $1 /\left(r_{l-1}\right)$ for problems in polar coordinates, which would require modification at the singular point $l=1$ since $r_{0}=0$. Also, these methods fail to compute if it is difficult to differentiate the singular coefficients twice. In this article, using 
Table 8 Example 8(a): Navier-Stokes' model equations (58.1)-(58.2) in spherical polar coordinates in $\boldsymbol{r} \boldsymbol{\theta} \boldsymbol{\theta}$ plane

\begin{tabular}{|c|c|c|c|c|c|c|c|}
\hline \multirow{2}{*}{\multicolumn{2}{|c|}{$\boldsymbol{h}$}} & \multicolumn{3}{|c|}{ Proposed $O\left(h^{4}\right)$-methods } & \multicolumn{3}{|c|}{$O\left(h^{4}\right)$-Methods discussed in $[16,17]$} \\
\hline & & $R_{e}=10$ & $R_{e}=100$ & $R_{e}=500$ & $R_{e}=10$ & $R_{e}=100$ & $R_{e}=500$ \\
\hline \multirow[t]{6}{*}{$1 / 4$} & u & $4.0350(-03)$ & $4.7668(-02)$ & $1.0430(-01)$ & $4.6880(-03)$ & $5.3210(-02)$ & $2.1678(-01)$ \\
\hline & $v$ & 2.8464(-03) & $2.4988(-02)$ & $8.0180(-02)$ & $3.6584(-03)$ & $3.0824(-02)$ & $8.8249(-02)$ \\
\hline & $u_{r}$ & $1.9901(-01)$ & $6.1644(-01)$ & $3.5356(+00)$ & $2.7257(-01)$ & $6.9552(-01)$ & $4.3682(+00)$ \\
\hline & $v_{r}$ & $4.8578(-02)$ & $2.7314(-01)$ & $2.0524(+00)$ & $5.6682(-02)$ & $3.5231(-01)$ & $2.8934(+00)$ \\
\hline & $u_{\theta}$ & $1.4393(-02)$ & $3.7298(-01)$ & $2.0017(+00)$ & $2.2692(-02)$ & $4.5332(-01)$ & $2.8126(+00)$ \\
\hline & $v_{\theta}$ & $1.1827(-02)$ & $1.9936(-01)$ & $1.0787(+00)$ & $1.9770(-02)$ & $2.7658(-01)$ & $1.8655(+00)$ \\
\hline \multirow[t]{6}{*}{$1 / 8$} & u & $4.5574(-04)$ & $6.9580(-03)$ & $2.4922(-02)$ & $5.0324(-04)$ & 7.3214(-03) & $2.9911(-02)$ \\
\hline & $v$ & $1.9624(-04)$ & $2.9897(-03)$ & $1.5191(-02)$ & $2.4454(-04)$ & $3.4789(-03)$ & $1.9923(-02)$ \\
\hline & $u_{r}$ & $5.2907(-02)$ & $8.0040(-02)$ & $3.8376(-01)$ & $5.7709(-02)$ & $8.5140(-02)$ & $4.3673(-01)$ \\
\hline & $v_{r}$ & $1.2219(-02)$ & $5.8063(-02)$ & $3.1326(-01)$ & $1.7912(-02)$ & $6.3360(-02)$ & $3.6623(-01)$ \\
\hline & $u_{\theta}$ & $1.7268(-03)$ & $3.4954(-02)$ & $2.1949(-01)$ & $2.2862(-03)$ & $3.9459(-02)$ & $2.6444(-01)$ \\
\hline & $v_{\theta}$ & $1.5710(-03)$ & $3.6024(-02)$ & $2.0974(-01)$ & $2.0017(-03)$ & $4.1420(-02)$ & $2.5479(-01)$ \\
\hline \multirow[t]{6}{*}{$1 / 16$} & u & $2.6553(-05)$ & $6.3235(-04)$ & $2.4796(-03)$ & $3.0355(-05)$ & $6.6532(-04)$ & $2.7697(-03)$ \\
\hline & v & $1.2965(-05)$ & $3.0081(-04)$ & $1.0288(-03)$ & $1.5569(-05)$ & $3.3180(-04)$ & $1.3288(-03)$ \\
\hline & $u_{r}$ & $1.5887(-02)$ & $1.5849(-02)$ & $3.2901(-02)$ & $1.9788(-02)$ & $1.8948(-02)$ & $3.5109(-02)$ \\
\hline & $v_{r}$ & $3.1718(-03)$ & $5.0558(-03)$ & $3.4203(-02)$ & $3.4817(-03)$ & $5.3855(-03)$ & $3.7302(-02)$ \\
\hline & $u_{\theta}$ & $1.2981(-04)$ & $3.3899(-03)$ & $2.5863(-02)$ & $1.5189(-04)$ & $3.6998(-03)$ & $2.8368(-02)$ \\
\hline & $v_{\theta}$ & $3.2230(-04)$ & $2.5193(-03)$ & $1.7076(-02)$ & $3.5032(-04)$ & $2.8391(-03)$ & $2.0670(-02)$ \\
\hline
\end{tabular}

Table 9 Example 8(b): Navier Stokes' model equations (59.1)-(59.2) in cylindrical polar coordinates in $\boldsymbol{r} \boldsymbol{\theta} \boldsymbol{\theta}$ plane

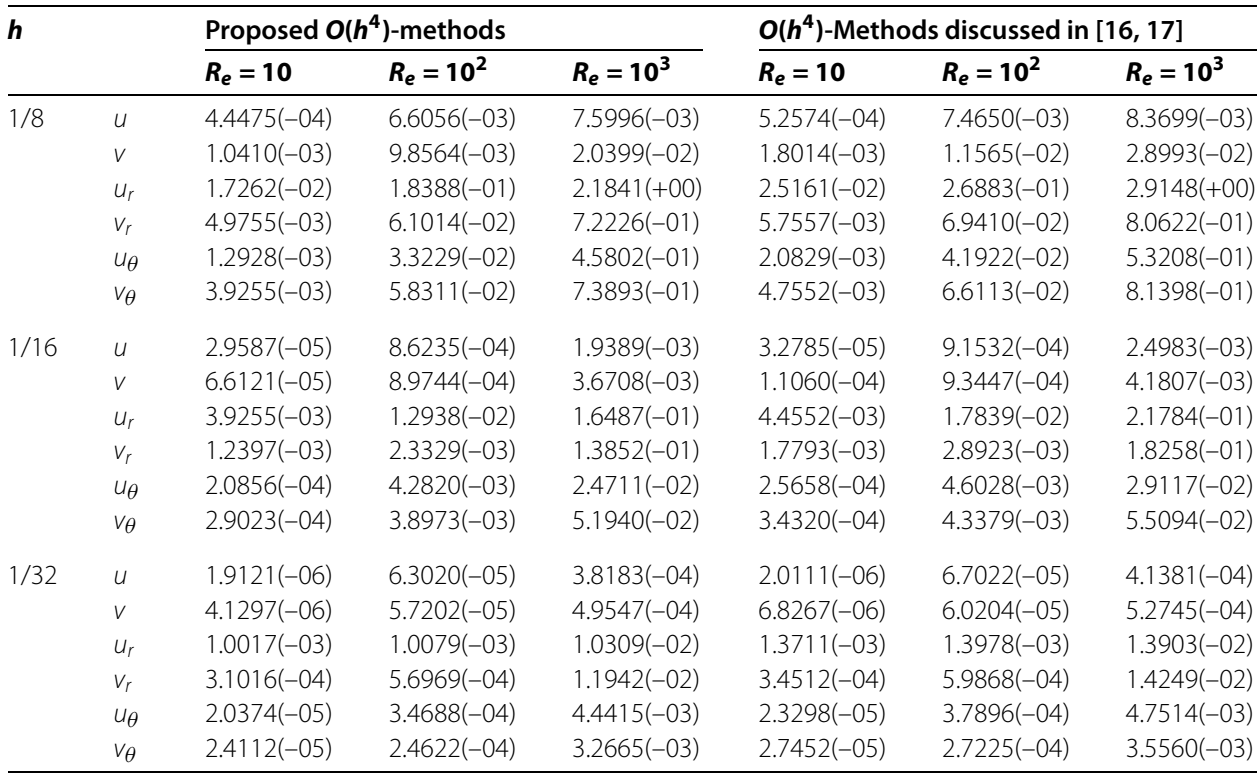

the same number of grid points, we have derived a new stable method of accuracy four, which instead involves the terms like $1 /\left(r_{l-1 / 2}\right)$, and hence is more convenient to implement at singular points. Thus, the proposed numerical method is directly applicable to an elliptic equation in polar coordinates, and we do not require any fictitious points for computation, which is the main highlight of our work. We have also derived fourth-order compact difference methods for the normal derivatives of the solution to the concerned problem. Also, we have compared our methods with the existing fourth-order numerical methods and found that our methods produce better results. 
Table 10 Example 8(c): Navier-Stokes' model equations (60.1)-(60.2) in cylindrical polar coordinates in $r$-z plane

\begin{tabular}{|c|c|c|c|c|c|c|c|}
\hline \multirow[t]{2}{*}{$\boldsymbol{h}$} & & \multicolumn{3}{|c|}{ Proposed $O\left(h^{4}\right)$-methods } & \multicolumn{3}{|c|}{$O\left(h^{4}\right)$-Methods discussed in $[16,17]$} \\
\hline & & $R_{e}=10$ & $R_{e}=10^{2}$ & $R_{e}=10^{3}$ & $R_{e}=10$ & $R_{e}=10^{2}$ & $R_{e}=10^{3}$ \\
\hline \multirow[t]{6}{*}{$1 / 8$} & $u$ & $1.2914(-04)$ & $1.1797(-03)$ & $1.1198(-03)$ & $2.0419(-04)$ & $1.9997(-03)$ & $1.9891(-03)$ \\
\hline & $v$ & $2.4545(-04)$ & $1.4028(-03)$ & $2.5592(-03)$ & $3.2445(-04)$ & $2.1820(-03)$ & $3.2295(-03)$ \\
\hline & $u_{r}$ & $6.7822(-03)$ & $5.7549(-02)$ & $5.6655(-01)$ & $7.5228(-03)$ & $6.5945(-02)$ & $6.4556(-01)$ \\
\hline & $v_{r}$ & $6.1035(-03)$ & $5.5251(-02)$ & $5.3119(-01)$ & $6.8530(-03)$ & $6.3152(-02)$ & $6.1911(-01)$ \\
\hline & $u_{z}$ & $4.7567(-04)$ & $8.1710(-03)$ & $8.9576(-02)$ & $5.5765(-04)$ & $8.8017(-03)$ & $9.6675(-02)$ \\
\hline & $v_{z}$ & $1.0385(-03)$ & $1.8142(-02)$ & $2.0159(-01)$ & $1.4583(-03)$ & $2.6241(-02)$ & $2.8951(-01)$ \\
\hline \multirow[t]{6}{*}{$1 / 16$} & u & $8.8980(-06)$ & $2.0869(-04)$ & $3.3219(-04)$ & $1.2192(-05)$ & $2.5968(-04)$ & $3.8912(-04)$ \\
\hline & $v$ & $1.4720(-05)$ & $1.6494(-04)$ & $4.2371(-04)$ & $1.9882(-05)$ & $2.1594(-04)$ & $4.7173(-04)$ \\
\hline & $u_{r}$ & $5.4068(-04)$ & $5.6217(-03)$ & $4.8410(-02)$ & $6.2860(-04)$ & $6.1712(-03)$ & $5.3014(-02)$ \\
\hline & $v_{r}$ & $5.1521(-04)$ & 4.6938(-03) & $4.4798(-02)$ & $5.6125(-04)$ & $5.1839(-03)$ & $4.9897(-02)$ \\
\hline & $u_{z}$ & $9.6347(-05)$ & $1.0593(-03)$ & 7.7984(-03) & $1.0743(-04)$ & $1.5395(-03)$ & $8.2489(-03)$ \\
\hline & $v_{z}$ & $8.3467(-05)$ & $1.1512(-03)$ & $1.5110(-02)$ & $8.8634(-05)$ & $1.6215(-03)$ & $2.0011(-02)$ \\
\hline \multirow[t]{6}{*}{$1 / 32$} & $u$ & $1.1626(-06)$ & $1.6762(-05)$ & $8.1323(-05)$ & $7.4808(-07)$ & $1.9267(-05)$ & $8.4323(-05)$ \\
\hline & v & $9.0095(-07)$ & $8.7775(-06)$ & $8.2463(-05)$ & $1.1469(-06)$ & $9.0577(-06)$ & $8.5364(-05)$ \\
\hline & $u_{r}$ & $1.1979(-04)$ & $5.7479(-04)$ & $5.8667(-03)$ & $1.3778(-04)$ & $6.0974(-04)$ & $6.1764(-03)$ \\
\hline & $v_{r}$ & $3.7573(-05)$ & $3.4598(-04)$ & $3.3449(-03)$ & $4.0375(-05)$ & $3.7895(-04)$ & $3.6947(-03)$ \\
\hline & $u_{z}$ & $1.9249(-05)$ & $1.7590(-04)$ & $4.3780(-04)$ & $2.2942(-05)$ & $2.0095(-04)$ & $4.6086(-04)$ \\
\hline & $v_{z}$ & $1.0944(-05)$ & $8.8478(-05)$ & $8.8297(-04)$ & $1.2448(-05)$ & $9.1874(-05)$ & $9.1792(-04)$ \\
\hline
\end{tabular}

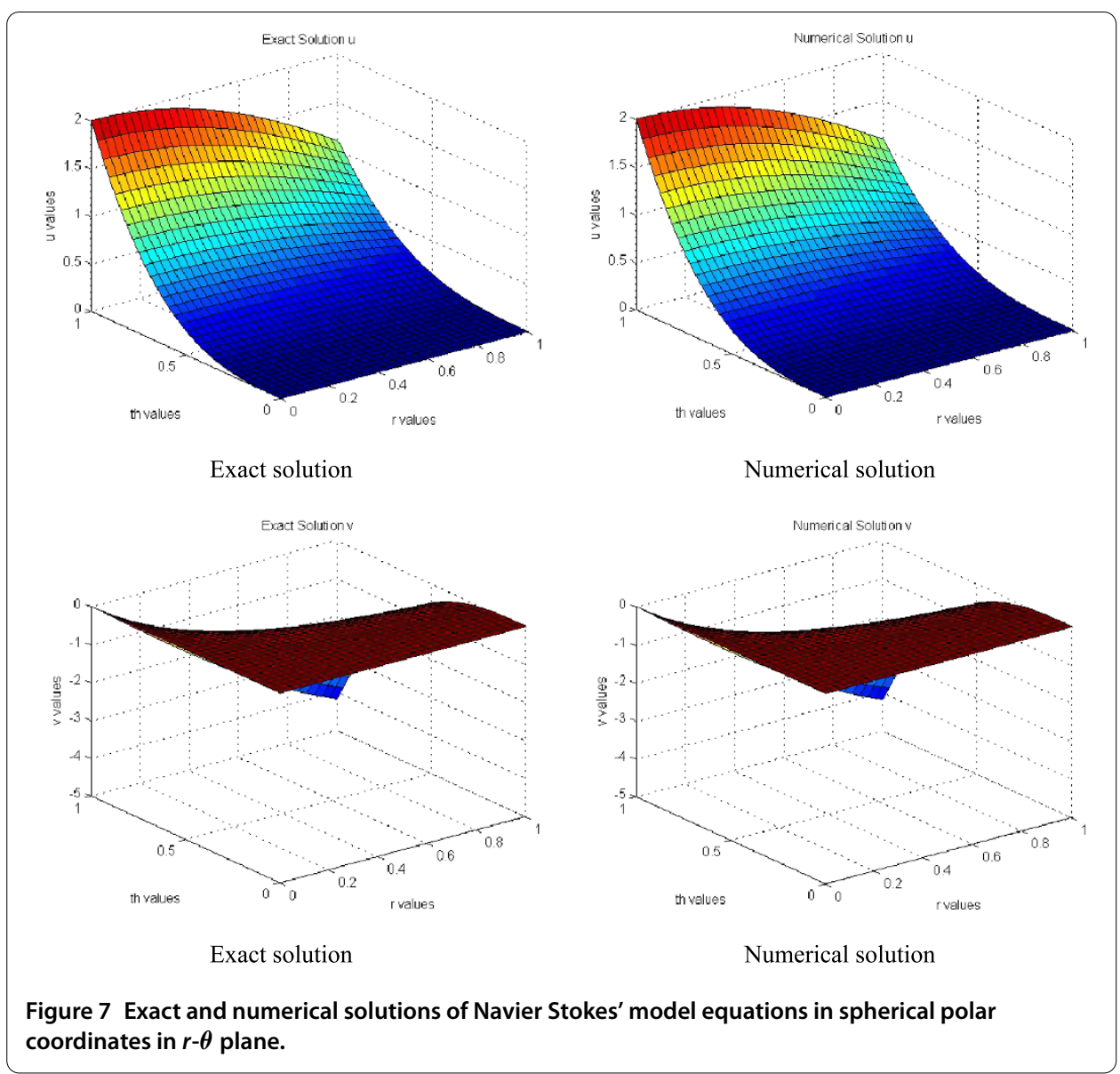




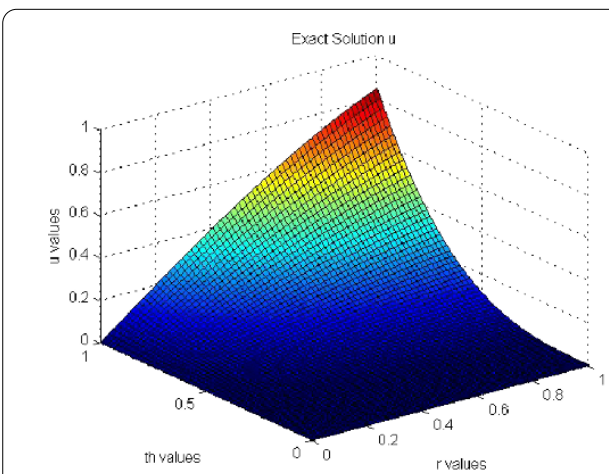

Exact solution $u$

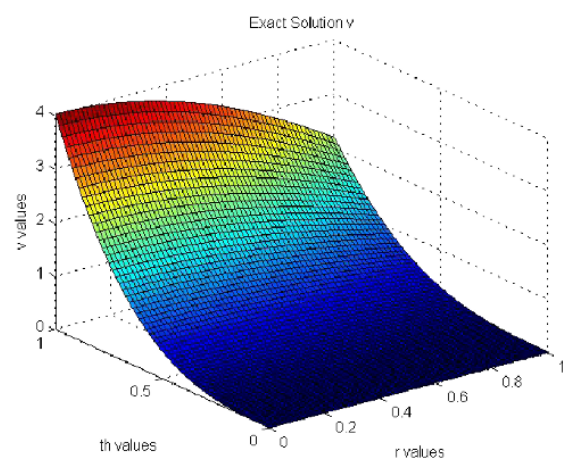

Exact solution $v$

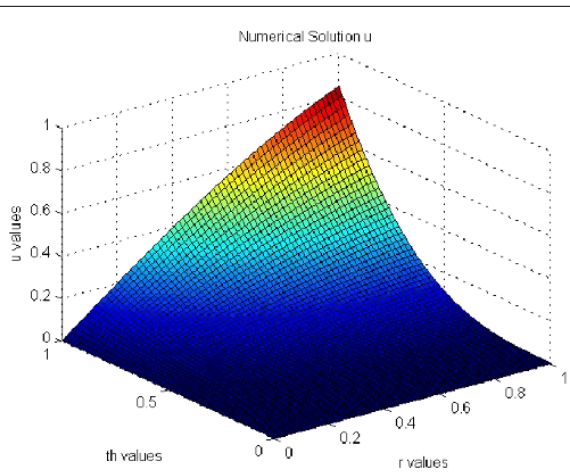

Numerical solution $u$

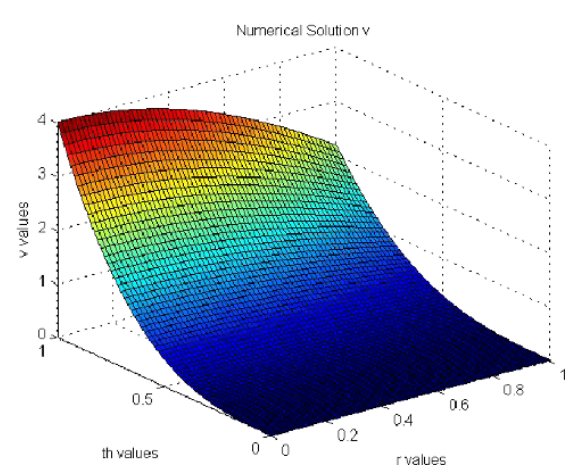

Numerical solution $v$

Figure 8 Exact and numerical solutions of Navier Stokes' model equations in cylindrical polar coordinates in $r-\theta$ plane. 


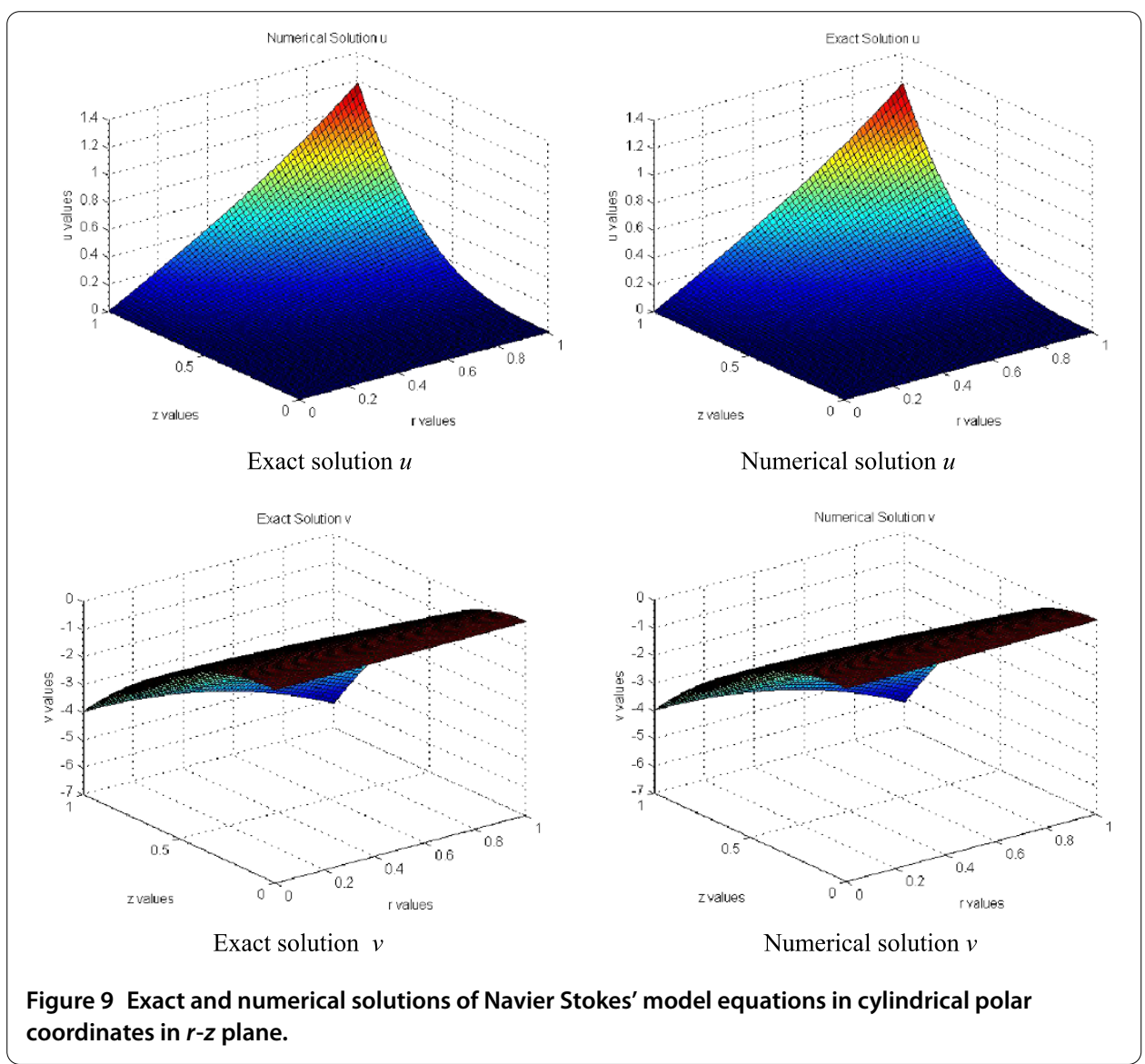




\section{Competing interests}

The authors declare that they have no competing interest.

\section{Authors' contributions}

RKM discussed the difference method based on off-step discretization. NS discussed the convergence analysis of the method and the application of the proposed method to a diffusion equation and its stability analysis. NS also carried out all the computational work. All authors read and approved the final manuscript.

\section{Author details}

'Department of Applied Mathematics, Faculty of Mathematics and Computer Science, South Asian University, Akbar Bhawan, Chanakyapuri, New Delhi, 110021, India. ²Department of Mathematics, Faculty of Mathematical Sciences, University of Delhi, Delhi, 110007, India.

\section{Acknowledgements}

This research was (i) partially supported by the South Asian University, and (ii) partially supported by the Council of Scientific and Industrial Research under research grant No. 09/045(0903)/2009-EMR-I. The authors thank the reviewers for their valuable suggestions, which substantially improved the standard of the paper.

Received: 15 March 2013 Accepted: 8 July 2013 Published: 24 July 2013

\section{References}

1. Jain, MK, Jain, RK, Mohanty, RK: Fourth order difference methods for the system of 2-D nonlinear elliptic partial differential equations. Numer. Methods Partial Differ. Equ. 7, 227-244 (1991)

2. Carey, GF: Computational Grids: Generation, Adaption and Solution Strategies. Taylor \& Francis, Washington, DC (1997)

3. Yavneh, IV: Analysis of a fourth-order compact scheme for convection diffusion. J. Comput. Phys. 133, $361-364$ (1997)

4. Zhang, J: On convergence and performance of iterative methods with fourth order compact schemes. Numer. Methods Partial Differ. Equ. 14, 263-280 (1998)

5. Zhang, J: On convergence of iterative methods for a fourth-order discretization scheme. Appl. Math. Lett. 10, 49-55 (1997)

6. Jain, MK, Jain, RK, Krishna, M: Fourth order difference method for quasi-linear Poisson equation in cylindrical symmetry. Commun. Numer. Methods Eng. 10, 291-296 (1994)

7. Gupta, MM: A fourth-order Poisson solver. J. Comput. Phys. 55, 166-172 (1984)

8. Gupta, MM, Manohar, RP, Stephenson, JW: A single cell high order scheme for the convection-diffusion equation with variable coefficients. Int. J. Numer. Methods Fluids 4, 641-651 (1984)

9. Spotz, WF, Carey, GF: High order compact scheme for the steady stream function vorticity equations. Int. J. Numer Methods Eng. 38, 3497-3512 (1995)

10. Ananthakrishnaiah, U, Saldanha, G: A fourth order finite difference scheme for two-dimensional nonlinear elliptic partial differential equations. Numer. Methods Partial Differ. Equ. 11, 33-40 (1995)

11. Saldanha, G: Technical note: A fourth order finite difference scheme for a system of 2D nonlinear elliptic partia differential equations. Numer. Methods Partial Differ. Equ. 17, 43-53 (2001)

12. Erturk, E, Gökcöl, C: Fourth-order compact formulation of Navier-Stokes equations and driven cavity flow at high Reynolds numbers. Int. J. Numer. Methods Fluids 50, 421-436 (2006)

13. Liu, J, Wang, C: A fourth order numerical method for the primitive equations formulated in mean vorticity. Commun. Comput. Phys. 4, 26-55 (2008)

14. Ito, K, Qiao, Z: A high order compact MAC finite difference scheme for the Stokes equations: augmented variable approach. J. Comput. Phys. 227, 8177-8190 (2008)

15. Li, M, Tang, T, Fornberg, B: A compact fourth-order finite difference scheme for the steady state incompressible Navier-Stokes equations. Int. J. Numer. Methods Fluids 20, 1137-1151 (1995)

16. Mohanty, RK: Order $h^{4}$ difference methods for a class of singular two-space dimensional elliptic boundary value problems. J. Comput. Appl. Math. 81, 229-247 (1997)

17. Mohanty, RK, Dey, S: A new finite difference discretization of order four for $(\partial u / \partial n)$ for two-dimensional quasi-linear elliptic boundary value problems. Int. J. Comput. Math. 76, 505-576 (2001)

18. Mohanty, RK, Singh, S: A new fourth order discretization for singularly perturbed two dimensional non-linear elliptic boundary value problems. Appl. Math. Comput. 175, 1400-1414 (2006)

19. Chawla, MM, Shivakumar, PN: An efficient finite difference method for two-point boundary value problems. Neural Parallel Sci. Comput. 4, 387-396 (1996)

20. Stephenson, JW: Single cell discretization of order two and four for biharmonic problems. J. Comput. Phys. 55, 65-80 (1984)

21. Varga, RS: Matrix Iterative Analysis. Springer, New York (2000)

22. Henrici, P: Discrete Variable Methods in Ordinary Differential Equations. Wiley, New York (1962)

23. Hageman, LA, Young, DM: Applied Iterative Methods. Dover, New York (2004)

24. Kelly, CT: Iterative Methods for Linear and Non-Linear Equations. SIAM, Philadelphia (1995)

25. Saad, Y: Iterative Methods for Sparse Linear Systems. SIAM, Philadelphia (2003)

doi:10.1186/1687-1847-2013-223

Cite this article as: Mohanty and Setia: A new compact high order off-step discretization for the system of 2D quasi-linear elliptic partial differential equations. Advances in Difference Equations 2013 2013:223. 